\title{
SYMPLECTIC QUOTIENTS HAVE SYMPLECTIC SINGULARITIES
}

\author{
HANS-CHRISTIAN HERBIG, GERALD W. SCHWARZ, AND CHRISTOPHER SEATON
}

\begin{abstract}
Let $K$ be a compact Lie group with complexification $G$, and let $V$ be a unitary $K$-module. We consider the real symplectic quotient $M_{0}$ at level 0 of the homogeneous quadratic moment map as well as the complex symplectic quotient, defined here as the complexification of $M_{0}$. We show that if $(V, G)$ is 3-large, a condition that holds generically, then the complex symplectic quotient has symplectic singularities and is graded Gorenstein. This in particular implies that the real symplectic quotient is graded Gorenstein. In the case that $K$ is a torus or $\mathrm{SU}_{2}$, we show that these results hold without the hypothesis that $(V, G)$ is 3-large.
\end{abstract}

\section{InTRODUCTION}

Let $K$ be a compact Lie group, let $V$ be a unitary $K$-module and let $G=K_{\mathbb{C}}$ denote the complexification of $K$. We denote the Lie algebras of $K$ and $G$ by $\mathfrak{k}$ and $\mathfrak{g}$, respectively. Let $J: V \rightarrow \mathfrak{k}^{*}$ be the associated homogeneous quadratic moment map. The real symplectic quotient at the zero level of $J$ is $M_{0}=J^{-1}(0) / K$. The complexification of $M_{0}$ is the corresponding complex symplectic quotient; see Definitions 2.3 and 2.5. One may equivalently begin with a complex reductive group $G$ and a $G$-module $V$ and then choose a maximal compact subgroup $K$ of $G$ as well as a Hermitian structure on $V$ with respect to which the $K$-action is unitary.

In this paper, we demonstrate that complex symplectic quotients are frequently symplectic varieties in the sense of [Bea00. This in particular implies that they are Gorenstein with rational singularities. Moreover, we demonstrate that they are graded Gorenstein, sometimes called strongly Gorenstein, meaning that they are Gorenstein with no degree shift in their canonical module. This implies that the Zariski closure of the real symplectic quotient $M_{0}$ is also graded Gorenstein. Note that $M_{0}$ is in a natural way a semialgebraic set, see Section 2.2 . Then $\mathbb{R}\left[M_{0}\right]$, the ring of real regular functions on $M_{0}$, is also graded Gorenstein.

In order to state our main result, let us first explain our notation. For a $G$ module $V$, we let $\mu=J \otimes_{\mathbb{R}} \mathbb{C}: V \otimes_{\mathbb{R}} \mathbb{C} \simeq V \oplus V^{*} \rightarrow \mathfrak{g}^{*}$ denote the complex moment map. Then $\mu(v, \xi)(A)=\xi(A(v))$ for $A \in \mathfrak{g}$ and $(v, \xi) \in V \oplus V^{*}$. See Definition 2.1 for the definitions of $k$-large, $k$-principal, and stable, properties of a $G$-module $(V, G)$, and note that $k$-large implies $(k-1)$-large. If $(V, G)$ is at least 1-large, then the complex symplectic quotient is given by the categorical quotient $\mu^{-1}(0) / / G$. Our main result is the following.

2010 Mathematics Subject Classification. Primary 53D20, 13A50; Secondary 20G20, 57S15, $13 \mathrm{H} 10$.

Key words and phrases. singular symplectic reduction, symplectic singularity, moment map, Hamiltonian action, graded Gorenstein.

C.S. was supported by the E.C. Ellett Professorship in Mathematics. 
Theorem 1.1. Suppose that $(V, G)$ is 3-large. Then the complex symplectic quotient $\mu^{-1}(0) / / G$ has symplectic singularities and is graded Gorenstein. In particular, $\mu^{-1}(0) / / G$ has rational singularities.

Recall that a complex variety $Y$ has rational singularities if it is normal and, for every resolution of singularities $f: X \rightarrow Y, R^{i} f_{*} \mathcal{O}_{X}=0$ for $i>0$; see KM98, Section 5.1].

For a fixed choice of $K$ and $G$, Theorem 1.1 implies that the complex symplectic quotient associated to "most" $G$-modules has symplectic singularities and is graded Gorenstein. For example, if $G$ is connected and semisimple, then all but finitely many $V$ such that $V^{G}=\{0\}$ and each irreducible component of $V$ is almost faithful (i.e. the kernel of the action is finite) are 3-large; see Section 3.3 In Section 4 we will also show that the conclusions of Theorem 1.1 hold if $(V, G)$ is 2-large with additional hypotheses.

When $(V, G)$ fails to be 1-large, there are different ways of realizing $\mu^{-1}(0) / / G$ as a scheme and hence different definitions of the complex symplectic quotient. It is known that there are examples of $(V, G)$ such that, by some such definitions, the complex symplectic quotient $\mu^{-1}(0) / / G$ fails to have symplectic singularities, see Bec10, BLLT17 and Sections 2.2 and 6 below. In this paper, we propose another definition of the complex symplectic quotient for which we expect Theorem 1.1 to hold more generally, though the proof will require very different techniques. Specifically, we define the complex symplectic quotient to be the complexification of the real quotient, $\operatorname{Spec}\left(\mathbb{R}\left[M_{0}\right] \otimes_{\mathbb{R}} \mathbb{C}\right)$, see Definition 2.5, corresponding to taking the real radical of $(J)$ before complexifying. Then for the few $(V, G)$ that fail to satisfy the hypotheses of Theorem 1.1 we make the following.

Conjecture 1.2. For all $(V, G)$, the (modified) complex symplectic quotient of Definition 2.5 has symplectic singularities and is graded Gorenstein.

Other authors define the complex symplectic quotient to be either $\operatorname{Spec}(\mathbb{C}[V \oplus$ $\left.\left.V^{*}\right]^{G} /(\mu)^{G}\right)$ or $\operatorname{Spec}\left(\mathbb{C}\left[V \oplus V^{*}\right]^{G} /(\sqrt{\mu})^{G}\right)$, which can fail to have symplectic singularities or even be reduced or Cohen-Macaulay. See Section 2.2 for a discussion of these definitions as well as examples where they do not coincide. Using our definition, we are not aware of any counterexamples to Conjecture 1.2. It is important to note that the three definitions yield the same scheme in the case of $k$-large representations for $k \geq 1$; see Lemma 2.8. Hence, as $(V, G)$ is assumed 3-large in Theorem 1.1, the notation $\mu^{-1}(0) / / G$ is unambiguous, and this result holds for any of the three definitions.

One piece of evidence in the direction of Conjecture 1.2 is related to the question of which (real) symplectic quotients are (graded regularly) symplectomorphic to a symplectic orbifold, the quotient of a real symplectic vector space by a finite group of symplectomorphisms. The authors have demonstrated in [HSS15, Theorem 1.3] that such isomorphisms are rare: one can only exist if $(V, G)$ fails to be 2-large (or even 2-principal and stable if $G$ is connected, HSS15, Theorem 1.1]). This has similar implications for the complex symplectic quotients; see Section 2 A symplectic orbifold has symplectic singularities by [Bea00, Proposition 2.4] and is graded Gorenstein by the work of Watanabe Wat74; see [Sta79, Theorem 7.1] and [HHS15, Section 6.1]. Hence, only symplectic quotients associated to "small" $G$ modules inherit the properties of being symplectic varieties and graded Gorenstein 
via an isomorphism with an orbifold, while Theorem 1.1 demonstrates these properties for "large" $G$-modules. A major part of the motivation of this investigation is the observation that symplectic quotients are rarely isomorphic to orbifolds yet appear to share many of the properties of orbifolds. While our emphasis is on "large" $G$-modules, the recent work of Bulois, Lehn, Lehn, and Terpereau [BLLT17] focuses on defining the complex symplectic quotients for "small" $G$-modules, specifically ones which are polar. For small cases, the three definitions of the complex symplectic quotient can yield different results, and the quotients considered in [BLLT17] are quite often orbifolds.

Other evidence for Conjecture 1.2] is as follows. In [HS14, Section 8.3], the Hilbert series of the ring of regular functions on the (real) symplectic quotients corresponding to several non-1-large representations of $\mathrm{O}_{n}(\mathbb{C})$ and $\mathrm{SU}_{2}$ were computed and seen to be so-called symplectic Hilbert series; this condition was later identified in [HHS15, Corollary 1.8] to be equivalent to the ring being graded Gorenstein. In CHS16, symplectic quotients corresponding to sums of the standard representations of $\mathrm{O}_{n}(\mathbb{C})$ and $\mathrm{SO}_{n}(\mathbb{C})$ were studied. For small cases, the graded Gorenstein property was verified by explicit computation of the Hilbert series. It was demonstrated that, among these representations, $\mu^{-1}(0)$ already has rational singularities if the representation $\left(V, \mathrm{SO}_{n}(\mathbb{C})\right)$ is 2-large. We will see other examples of $(V, G)$ such that $\mu^{-1}(0)$ has rational singularities; see Proposition 5.4 and Section 6 .

The complex symplectic quotients corresponding to classical representations of $\mathrm{GL}_{n}(\mathbb{C}), \mathrm{O}_{n}(\mathbb{C})$, and $\mathrm{Sp}_{n}(\mathbb{C})$ have been shown to have symplectic singularities in [Ter12, Appendix A.2], see also [BLLT17, Ter14. Becker [Bec10] has demonstrated that the complex symplectic quotients associated to certain representations of $\mathrm{SL}_{2}(\mathbb{C})$ are symplectic varieties as well. Note that these references consider also the construction of symplectic resolutions of these symplectic quotients. The existence of symplectic resolutions appears to be rare among symplectic varieties; see Ver00, Theorem 3.2]. Bellamy and Schedler BS16, have demonstrated that Nakajima quiver varieties are symplectic varieties and identified which admit symplectic resolutions. More recently and using the results contained in this paper, they have shown in BS18 that if $(V, G)$ is 3-large, $G /[G, G]$ is finite, and $G$ acts nontrivially on $V$, then the complex symplectic quotient does not admit a symplectic resolution. They have further demonstrated that for $(V, G) 3$-large, the complex symplectic quotient has terminal singularities, is $\mathbb{Q}$-factorial if and only if $G /[G, G]$ is finite, and is locally factorial when $G /[G, G]$ is trivial. As explained above, the definition of complex symplectic quotient is different than ours in the above references, though the definitions coincide in 1-large cases.

To further illustrate Conjecture 1.2, we consider non-3-large $G$-modules for certain $G$. We first consider the case of a torus, yielding the following.

Theorem 1.3. Assume the identity component $K^{0}$ of $K$ is a torus. Then the corresponding (modified) complex symplectic quotient of Definition 2.5 has symplectic singularities and is graded Gorenstein. In particular, the complex symplectic quotient has rational singularities.

Note that in the case that $(V, K)$ is unimodular, meaning that all maximal minors of the weight matrix of the action are \pm 1 or 0 , Theorem 1.3 was established in [BK12, Proposition 4.11]. See also [Bul18, Section 3], where it is demonstrated that when $G$ is a torus, the alternate definition given in Equation (2.5) yields complex symplectic quotients that are in general reduced and normal. 
Similarly, for $G=\mathrm{SL}_{2}(\mathbb{C})$, we will indicate that Theorem 1.1 applies as well when $(V, G)$ is 2-large. The few $G$-modules that fail to be 2-large have been determined in Sch95, Theorem 11.9], and many of the corresponding symplectic quotients were found to be isomorphic to linear symplectic orbifolds in HSS15, Theorem 1.6]. Considering the remaining few, we obtain the following.

Theorem 1.4. Let $K=\mathrm{SU}_{2}$ and let $(V, K)$ be unitary. Then the the corresponding (modified) complex symplectic quotient of Definition [2.5 has symplectic singularities and is graded Gorenstein. In particular, the complex symplectic quotient has rational singularities.

The outline of this paper is as follows. In Section 2, we recall the relevant background information we need. Section 2.1 gives definitions of the hypotheses for $G$-modules that will play a role in this work, Section 2.2 explains our definition of the real and complex symplectic quotients, and Section 2.3 recalls the background and results on symplectic and Gorenstein singularities. In Section 3 we establish several auxiliary results we will need about the structure of $\mu^{-1}(0)$ and the properties of $G$-modules. In particular, in Section 3.4 we give a symplectic slice theorem for complex symplectic quotients similar to that of [BLLT17, Section 6] and use it to demonstrate that for many $G$-modules, the complex symplectic quotient inherits a symplectic structure on its smooth locus; see Corollary 3.18. We then prove Theorem 1.1 in Section 4 In Section 5 we consider the case of $G^{0}$ a torus and prove Theorem 1.3 while in Section [6. we consider the case of $K=\mathrm{SU}_{2}$ and prove Theorem 1.4 .

\section{ACKNOWLEDGEMENTS}

The authors would like to thank Arnaud Beauville, William Fulton, and Hanspeter Kraft for helpful responses to our questions as well as Michaël Bulois for helpful comments and references and pointing out an error in an earlier draft of this paper. We would also like to thank the referees for a careful reading of the manuscript and for many suggestions that improved this paper.

\section{BACKGROUND AND DEFINITIONS}

2.1. Properties of $(V, G)$. Throughout this paper, $K$ will denote a compact Lie group and $G=K_{\mathbb{C}}$ its complexification, i.e., $G$ is a complex reductive group and $K$ is a maximal compact subgroup of $G$. Let $V$ be a $G$-module. The categorical quotient $V / / G=\operatorname{Spec}\left(\mathbb{C}[V]^{G}\right)$ is an affine variety that parameterizes the closed orbits in $G$. If $G v$ is a closed orbit, then the isotropy group $G_{v}$ is reductive by Matsushima's theorem Mat60, Lun72, and the isotropy type of the orbit $G v$ is the conjugacy class of $G_{v}$. The variety $V / / G$ is stratified by isotropy type [un73], and there is a unique open stratum $(V / / G)_{\mathrm{pr}}$ called the principal stratum; the isotropy groups of points in the corresponding closed orbits are called principal isotropy groups. We let $V_{\mathrm{pr}}=\pi^{-1}\left((V / / G)_{\mathrm{pr}}\right)$ where $\pi: V \rightarrow V / / G$ denotes the orbit map, the map dual to the inclusion $\mathbb{C}[V]^{G} \subset \mathbb{C}[V]$. We say that a subset of $V$ is $G$ saturated if it is a union of fibers of $\pi$.

Definition 2.1. Let $V$ be a $G$-module. We say that $(V, G)$ has $F P I G$ if the principal isotropy groups are finite and $T P I G$ if they are trivial. We say that $(V, G)$ is stable if there is an open set of closed orbits; equivalently, if $V_{\mathrm{pr}}$ consists of closed orbits. For $k \geq 1$, we say that $(V, G)$ is $k$-principal if $\operatorname{codim}_{\mathbb{C}} V \backslash V_{\mathrm{pr}} \geq k$. Letting $V_{(r)}$ 
denote the locally closed set of points with isotropy group of complex dimension $r$, $(V, G)$ is $k$-modular if $\operatorname{codim}_{\mathbb{C}} V_{(r)} \geq r+k$ for $1 \leq r \leq \operatorname{dim}_{\mathbb{C}} G$. Note in particular that if $(V, G)$ is $k$-modular for $k \geq 0$, then $V_{(0)} \neq \emptyset$. Finally, $(V, G)$ is $k$-large if it is $k$-principal, $k$-modular, and has FPIG.

See Sch95 for more background on these concepts, and note that references sometimes differ about whether FPIG is required as part of the definition of $k$ modular or $k$-principal.

Applying the definition of $k$-modular to $V^{G}$, we have the following.

Lemma 2.2. Let $V$ be a $k$-modular $G$-module where $G$ is not finite and $k \geq 0$. Then $\operatorname{dim}_{\mathbb{C}} G+k \leq \operatorname{dim}_{\mathbb{C}} V-\operatorname{dim}_{\mathbb{C}} V^{G}$.

Proof. As $V^{G} \subset V_{\left(\operatorname{dim}_{\mathbb{C}} G\right)}$, we have $\operatorname{dim}_{\mathbb{C}} V^{G} \leq \operatorname{dim}_{\mathbb{C}} V_{\left(\operatorname{dim}_{\mathbb{C}} G\right)} \leq \operatorname{dim}_{\mathbb{C}} V-\operatorname{dim}_{\mathbb{C}} G-$ $k$.

2.2. Real and complex symplectic quotients. For a unitary $K$-module $V$ with $\operatorname{dim}_{\mathbb{C}} V=n$, let $z_{1}, \ldots, z_{n}$ be a choice of unitary coordinates for $V$. Considering $V$ with its underlying structure as a $2 n$-dimensional real vector space, we equip $V$ with the symplectic form

$$
\omega=\omega_{V}=\frac{\sqrt{-1}}{2} \sum_{j=1}^{n} d z_{j} \wedge d \bar{z}_{j}
$$

and then the corresponding Poisson bracket on $\mathcal{C}^{\infty}(V)$ satisfies

$$
\left\{z_{i}, \bar{z}_{j}\right\}=\frac{2}{\sqrt{-1}} \delta_{i, j}, \quad\left\{z_{i}, z_{j}\right\}=\left\{\bar{z}_{i}, \bar{z}_{j}\right\}=0 .
$$

The action of $K$ on $V$ is Hamiltonian with moment map $J: V \rightarrow \mathfrak{k}^{*}$ defined by

$$
\langle J(v), A\rangle=\frac{\sqrt{-1}}{2}\langle v, A v\rangle, \quad v \in V, A \in \mathfrak{k} .
$$

Choosing a basis $A_{1}, \ldots, A_{m}$ for $\mathfrak{k}$, the components $J_{i}:=\left\langle J(\cdot), A_{i}\right\rangle$ are homogeneous quadratic elements of the algebra $\mathbb{R}[V]$ of real regular functions on $V$. We let $(J)$ denote the ideal generated by the $J_{i}$ in $\mathbb{R}[V]$ and let $M$ denote the zero set of $J$. Let $\mathbb{R}[V]^{K}$ denote the $K$-invariant polynomials on $V$, a finitely generated graded algebra Wey39, Chapter 8, Section 14], and let $(J)^{K}:=(J) \cap \mathbb{R}[V]^{K}$ denote the invariant part of $(J)$.

Definition 2.3. Let $K$ be a compact Lie group and $V$ a unitary $K$-module. Let $J: V \rightarrow \mathfrak{k}^{*}$ denote the associated homogeneous quadratic moment map. The (real) shell associated to $(V, K)$ is the set $M$ with ideal $\sqrt[\mathbb{R}]{(J)}$, the real radical of $J$, which is the same thing as the ideal of $\mathbb{R}[V]$ vanishing on $M$. The real symplectic quotient associated to $(V, K)$ is the quotient $M_{0}:=M / K$. Thus the Poisson algebra of real regular functions on the real symplectic quotient is defined by

$$
\mathbb{R}\left[M_{0}\right]:=\mathbb{R}[V]^{K} /(\sqrt[\mathbb{R}]{(J)})^{K}
$$

where $(\sqrt[\mathbb{R}]{(J)})^{K}:=\sqrt[\mathbb{R}]{(J)} \cap \mathbb{R}[V]^{K}$.

Note that as $J$ is equivariant, the Poisson bracket on $\mathbb{R}[V]$ induces a well-defined Poisson bracket on $\mathbb{R}\left[M_{0}\right]$. The real symplectic quotient is a symplectic stratified space [SL91] and is a semialgebraic subset of the semialgebraic set $V / K$ [PS85]. See also AGJ90, FHS13, HS13, HS14 for background on real symplectic quotients and their algebra of real regular functions. 
Remark 2.4. It is frequently the case that $J$ generates a real ideal of $\mathbb{R}[V]$, i.e., $\sqrt[\mathbb{R}]{(J)}=(J)$. Recalling that $G=K_{\mathbb{C}}$, this is in particular true if $(V, G)$ is 1-large by HS13, Corollary 4.3]. Even if this condition fails, it can happen that the invariant part of the real radical of $(J)$ is the invariant part of $(J)$, i.e. $(\sqrt[\mathbb{R}]{(J)})^{K}=(J)^{K}$; see Example 2.9 below. In these cases, we of course have

$$
\mathbb{R}\left[M_{0}\right]=\mathbb{R}[V]^{K} /(J)^{K} .
$$

The action of $K$ on $V$ extends to an action of $G$ on $V$. We complexify the real vector space $V$ to form $V_{\mathbb{C}}:=V \otimes_{\mathbb{R}} \mathbb{C}$, which is isomorphic as a $K$-module to $V \oplus V^{*}$, and we take the $G$-action to be the obvious one on $V \oplus V^{*}$. Letting $\left(w_{1}, \ldots, w_{n}\right)$ denote coordinates dual to $\left(z_{1}, \ldots, z_{n}\right)$, we equip $V \oplus V^{*}$ with the complex symplectic form

$$
\omega=\omega_{V_{\mathbb{C}}}=\sum_{j=1}^{n} d z_{j} \wedge d w_{j} .
$$

The action of $G$ on $V \oplus V^{*}$ is Hamiltonian with moment map $\mu=J \otimes_{\mathbb{R}} \mathbb{C}: V \oplus V^{*} \rightarrow$ $\mathfrak{g}^{*}$, the complexification of the real moment map, which is given by $\mu(v, \xi)(A)=$ $\xi(A(v))$ for $A \in \mathfrak{g}$ and $(v, \xi) \in V \oplus V^{*}$, see [HS13. Section 4]. The (complex) shell $N$ associated to $(V, G)$ is the subscheme of $V \oplus V^{*}$ associated to the ideal $(\mu)$ generated by the components of $\mu$. With respect to a basis for $\mathfrak{g}$, we let $\mu_{i}$ denote the corresponding coordinates of $\mu$, and then the $\mu_{i}$ are homogeneous quadratic elements of the regular functions $\mathbb{C}\left[V \oplus V^{*}\right]$. Note that if the chosen basis consists of real vectors so that it is also a basis for $\mathfrak{k}$, then $\mu_{i}=J_{i} \otimes_{\mathbb{R}} \mathbb{C}$ for each $i$. We define the complex symplectic quotient to be the complexification of $M_{0}$ as follows.

Definition 2.5. Let $K$ be a compact Lie group, $V$ a unitary $K$-module and $G=$ $K_{\mathbb{C}}$. Let $\mu: V \oplus V^{*} \rightarrow \mathfrak{g}^{*}$ denote the moment map associated to the Hamiltonian action of $G$ on $V \oplus V^{*}$ with respect to its standard symplectic structure, i.e., $\mu=J \otimes_{\mathbb{R}} \mathbb{C}$. The complex symplectic quotient associated to $(V, G)$ is the complex algebraic variety

$$
\operatorname{Spec}\left(\mathbb{R}\left[M_{0}\right] \otimes_{\mathbb{R}} \mathbb{C}\right) .
$$

The Poisson bracket on $\mathbb{R}\left[M_{0}\right] \otimes_{\mathbb{R}} \mathbb{C}$ is that inherited from the bracket on $\mathbb{C}\left[V \oplus V^{*}\right]$ so that

$$
\left\{z_{i}, w_{j}\right\}=\frac{2}{\sqrt{-1}} \delta_{i, j}, \quad\left\{z_{i}, z_{j}\right\}=\left\{w_{i}, w_{j}\right\}=0
$$

where the $z_{i}$ are coordinates for $V$ and $w_{i}$ the dual coordinates for $V^{*}$.

This definition of complex symplectic quotient is not standard. Some authors consider the alternative quotient

$$
\operatorname{Spec}\left(\mathbb{C}\left[V \oplus V^{*}\right]^{G} /(\mu)^{G}\right),
$$

while others compute the radical in the complex sense and consider

$$
N / / G=\operatorname{Spec}\left(\mathbb{C}\left[V \oplus V^{*}\right]^{G} /(\sqrt{(\mu)})^{G}\right) .
$$

Definition 2.5, on the other hand, corresponds to computing the real symplectic reduction and then complexifying. More precisely, using Sch80, Proposition 5.8(1)], the variety of Equation (2.2) is

$$
N^{\prime} / / G=\operatorname{Spec}\left(\mathbb{C}\left[V \oplus V^{*}\right]^{G} /\left(\sqrt[\mathbb{R}]{(J)} \otimes_{\mathbb{R}} \mathbb{C}\right)^{G}\right),
$$


where $N^{\prime}$ is the variety of $\sqrt[\mathbb{R}]{(J)} \otimes_{\mathbb{R}} \mathbb{C}$ in $V \oplus V^{*}$, the complexification of the real shell. Note that in general, $\sqrt{(\mu)} \subset \sqrt[\mathbb{R}]{(J)} \otimes_{\mathbb{R}} \mathbb{C}$, and this inclusion may be strict; see Example 2.7 It follows that $\sqrt{(\mu)}^{G} \subset \sqrt[\mathbb{R}]{(J)}^{G} \otimes_{\mathbb{R}} \mathbb{C}$. It can happen that $\sqrt{(\mu)} \neq \sqrt[\mathbb{R}]{(J)} \otimes_{\mathbb{R}} \mathbb{C}$ while $\sqrt{(\mu)}^{G}=\sqrt[\mathbb{R}]{(J)}^{G} \otimes_{\mathbb{R}} \mathbb{C}$; see Examples 2.6 and 2.9 below. Our motivation for using Definition 2.5 is two-fold. First, we are primarily interested in using the complex symplectic quotient to study properties of the real quotient $M_{0}$ and its ring $\mathbb{R}\left[M_{0}\right]$ of real regular functions. Secondly, there are cases where the quotients defined by Equations (2.4) and (2.5) can be pathological in ways that do not appear to occur for complex symplectic quotients as defined in Definition 2.5. For example, Theorem 1.4 fails using either quotient given in Equations (2.4) and (2.5). We illustrate this fact and the differences between these three definitions with the following.

Example 2.6. Let $G=\mathrm{SL}_{2}(\mathbb{C})$, and let $V=R_{1}=\mathbb{C}^{2}$ denote the defining representation of $G$ on binary forms of degree 1. In standard coordinates $\left(z_{1}, z_{2}, w_{1}, w_{2}\right)$ for $V \oplus V^{*}$, the components of the moment map are given by $\mu_{1}=z_{1} w_{2}, \mu_{2}=z_{2} w_{1}$, and $\mu_{3}=z_{1} w_{1}-z_{2} w_{2}$. In this case, $(\mu)$ is not radical and hence $(J)$ is not real. However, the invariant part of the radical of $(\mu)$ is the augmentation ideal in the invariant ring $\mathbb{C}\left[V \oplus V^{*}\right]^{\mathrm{SL}_{2}(\mathbb{C})}$, and the invariant part of the real radical of $(J)$ is the augmentation ideal in $\mathbb{R}[V]^{K}$. Hence, $\mathbb{R}\left[M_{0}\right]=\mathbb{R}[V]^{K} /(\sqrt[\mathbb{R}]{(J)})^{K}=\mathbb{R}$ and $\mathbb{R}\left[M_{0}\right] \otimes_{\mathbb{R}} \mathbb{C}=\mathbb{C}$ so that the real and complex symplectic quotients are both points. See [AGJ90, Example 7.12] for a careful discussion.

In [Bec10, Section 3], Becker considers the complex symplectic quotient associated $\left(R_{1}, \mathrm{SL}_{2}(\mathbb{C})\right)$ using the definition of Equation (2.4). It is demonstrated that this quotient is not a reduced scheme and hence not a symplectic variety. In particular,

$$
\left[\mathbb{C}\left[V \oplus V^{*}\right] /(\mu)\right]^{\mathrm{SL}_{2}(\mathbb{C})}=\mathbb{C}\left[V \oplus V^{*}\right]^{\mathrm{SL}_{2}(\mathbb{C})} /(\mu)^{\mathrm{SL}_{2}(\mathbb{C})}=\mathbb{C}[z] /\left(z^{2}\right) .
$$

Becker corrects for this by equipping the quotient Spec $\left(\mathbb{C}[z] /\left(z^{2}\right)\right)$ with its reduced structure, yielding a point. Using the quotient in Equation (2.5), one obtains

$$
\left[\mathbb{C}\left[V \oplus V^{*}\right] / \sqrt{(\mu)}\right]^{\mathrm{SL}_{2}(\mathbb{C})}=\mathbb{C}\left[V \oplus V^{*}\right]^{\mathrm{SL}_{2}(\mathbb{C})} /(\sqrt{(\mu)})^{\mathrm{SL}_{2}(\mathbb{C})}=\mathbb{C},
$$

which also yields a point and coincides with our Definition 2.5 of the complex symplectic quotient in this case.

Example 2.7. Let $G=\mathrm{SL}_{2}(\mathbb{C})$ and $V=R_{1}^{\oplus 2}$ using the notation of Example 2.6. The ideal $(\mu)$ is radical, but the ideal $(J)$ is not real; see AGJ90, Example 7.13]. In that reference, the real radical $\sqrt[\mathbb{R}]{(J)}$ of $(J)$ is computed, and it is shown ([AGJ90, Examples 5.11(a) and 7.6]) that the corresponding real symplectic quotient $M_{0}$ is isomorphic to the orbifold $\mathbb{C} /( \pm 1)$, and hence the complex symplectic quotient is given by $\mathbb{C}\left[p_{1}, p_{2}, p_{3}\right] /\left(p_{1} p_{2}-p_{3}^{2}\right)$. This can also be demonstrated using the fact that, for $V=R_{1}^{\oplus 2}$, the quotient $V / / \mathrm{SL}_{2}(\mathbb{C})$ is 1-dimensional over $\mathbb{C}$ so that $V$ is polar; see [HSS15, Section 5].

In this case, however, because $(\mu)$ is radical, the quotient defined in Equation (2.4) is isomorphic to the quotient defined in Equation (2.5) yet does not coincide with our Definition 2.5 of the complex symplectic quotient. Moreover, these alternative quotients satisfy none of the consequences of Theorem 1.1. In particular, using the explicit description of the moment map and invariants given in AGJ90. 
Example 7.13], one computes that $\mathbb{C}\left[V \oplus V^{*}\right]^{\mathrm{SL}_{2}(\mathbb{C})} /(\mu)^{\mathrm{SL}_{2}(\mathbb{C})}$ is generated by six quadratic polynomials $\sigma_{i}, i=1, \ldots, 6$ subject to the eleven relations

$$
\begin{aligned}
& \sigma_{3} \sigma_{6}, \quad \sigma_{2} \sigma_{6}, \quad \sigma_{1} \sigma_{6}, \quad \sigma_{3} \sigma_{5}, \quad \sigma_{2} \sigma_{5}, \quad \sigma_{1} \sigma_{5}, \\
& \sigma_{4}^{2}+\sigma_{5}^{2}+\sigma_{6}^{2}, \quad \sigma_{3} \sigma_{4}, \quad \sigma_{2} \sigma_{4}, \quad \sigma_{1} \sigma_{4}, \quad \sigma_{1}^{2}-\sigma_{2}^{2}-\sigma_{3}^{2} .
\end{aligned}
$$

This ring has depth 1 and Krull dimension 2, so the alternate quotient fails even to be Cohen-Macaulay. See [BLLT17, Example 8.7(2)].

We would like to stress, however, that these three notions of complex symplectic quotients do coincide for almost all representations. Specifically, we have the following.

Lemma 2.8. Let $(V, K)$ be unitary and let $G=K_{\mathbb{C}}$. If $J$ generates a real ideal of $\mathbb{R}[V]$, then

$$
\mathbb{R}\left[M_{0}\right] \otimes_{\mathbb{R}} \mathbb{C} \simeq \mathbb{C}\left[V \oplus V^{*}\right]^{G} /(\sqrt{\mu})^{G}=\mathbb{C}\left[V \oplus V^{*}\right]^{G} /(\mu)^{G} .
$$

In particular, this holds if $(V, G)$ is 1-large.

Proof. By [Sch80, Proposition 5.8(1)], we have

$$
\mathbb{C}\left[V \oplus V^{*}\right]^{G}=\mathbb{C}\left[V \oplus V^{*}\right]^{K} \simeq \mathbb{R}[V]^{K} \otimes_{\mathbb{R}} \mathbb{C} .
$$

If $J$ generates a real ideal in $\mathbb{R}[V]$, then $\mu=J \otimes_{\mathbb{R}} \mathbb{C}$ generates a radical ideal in $\mathbb{C}\left[V \oplus V^{*}\right]$ by AGJ90, Theorem 6.5]. Since $(\mu)$ is $G$-stable, $(\mu)^{G}:=(\mu) \cap \mathbb{C}\left[V \oplus V^{*}\right]^{G}$ is radical in $\mathbb{C}\left[V \oplus V^{*}\right]^{G}$. Hence

$$
\mathbb{R}\left[M_{0}\right] \otimes_{\mathbb{R}} \mathbb{C} \simeq\left(\mathbb{R}[V]^{K} \otimes_{\mathbb{R}} \mathbb{C}\right) /\left((J)^{K} \otimes_{\mathbb{R}} \mathbb{C}\right) \simeq \mathbb{C}\left[V \oplus V^{*}\right]^{G} /(\mu)^{G} .
$$

That the hypotheses hold when $(V, G)$ is 1-large is a consequence of HS13, Corollary 4.3] as noted in Remark 2.4.

Even in the rare cases that $(V, G)$ fails to be 1-large, it can happen that Equations (2.4) and (2.5) coincide with our definition of the complex symplectic quotient. For instance, there are non-1-large representations for which $J$ generates a real ideal, including the standard action of $\mathrm{O}_{n}(\mathbb{C})$ or $\mathrm{SO}_{n}(\mathbb{C})$ on $V=\mathbb{C}^{n}$ with $n \geq 3$; see AGJ90, Example 7.10]. However, even if the ideal generated by $J$ fails to be real, it can happen that $(J)^{K}=(\sqrt[\mathbb{R}]{(J)})^{K}$, as illustrated by the following.

Example 2.9. Let $V=\mathbb{C}$ with $G=\mathbb{C}^{\times}$-action given by multiplication. Then the only closed orbit is $\{0\}$ so that $(V, G)$ fails to be stable so cannot be 1 -large. The $G$-action on $V \oplus V^{*}=\mathbb{C}^{2}$ has weight vector $(1,-1)$. In real coordinates $(z, \bar{z})$ for $V$, the $K=\mathbb{S}^{1}$-moment map $J$ is, up to a scalar, $z \bar{z}=|z|^{2}$. The corresponding ideal $(J)=(z \bar{z})$ is clearly not real, as its zero set is the origin and its real radical contains $z$ and $\bar{z}$. On the other hand, using coordinates $(z, w)$ for $V \oplus V^{*}$, the complex moment map $\mu=z w$ generates the radical ideal $(z w)$.

However, the invariants of this action are generated by $z \bar{z}$ in the real algebra $\mathbb{R}[V]$ and $z w$ in the complex algebra $\mathbb{C}\left[V \oplus V^{*}\right]$. Therefore,

$$
(J)^{K}=(\sqrt[\mathbb{R}]{(J)})^{K}=J \cdot \mathbb{R}[J],
$$

and

$$
(\mu)^{G}=(\sqrt{(\mu)})^{G}=\mu \cdot \mathbb{C}[\mu] .
$$

Hence, similar to the case of Example2.6, the real and complex symplectic quotients are both a point. 
As another large family of examples, the closure $\overline{\mathcal{O}}$ of a nilpotent coadjoint orbit $\mathcal{O}$ by one of the classical Lie groups $\mathrm{GL}_{n}(\mathbb{C}), \mathrm{O}_{n}(\mathbb{C})$, or $\mathrm{SP}_{n}(\mathbb{C})$ has been shown by Kraft and Procesi KP79, KP82 to be isomorphic to what turns out to be a complex symplectic quotient using the definition in Equation (2.5); see [Bry03, Section 4]. Though $\overline{\mathcal{O}}$ may fail to be normal, its normalization has symplectic singularities by Pan91, see also [Fu03. Hence, the corresponding complex symplectic quotients via Equation (2.4) have symplectic singularities if and only if they are normal. The non-normal cases are associated to representations that must fail to be 2-modular, see Proposition 3.2 (iii); in some cases, the orbit closures are reducible and hence correspond to representations that cannot be 1-modular by Proposition 3.2 (ii), see KP82, 14.2]. Therefore, the complex symplectic quotients given by Equation (2.4) and Definition 2.5 may not coincide; whether they do has yet to be determined and will be considered elsewhere.

Remark 2.10. If $(V, G)$ is 1-large, then $M$ is Zariski dense in $N$ by HS13, Corollary 4.2]. If $(V, G)$ fails to be 1-large, then it is possible that $\operatorname{dim}_{\mathbb{R}} M<\operatorname{dim}_{\mathbb{C}} N$ as illustrated in Example 2.9. If $H$ is the isotropy group of a $K$-orbit in $M$, then $H_{\mathbb{C}}$ is the isotropy group of the corresponding closed $G$-orbit in $N$. However, the conjugacy classes of such isotropy groups do not necessarily exhaust those in $N$, as the following example shows.

Example 2.11. Let $K=\mathrm{SU}_{n}, G=\mathrm{SL}_{n}(\mathbb{C})$ and $V=\left(\mathbb{C}^{n}\right)^{\oplus 2 n}$ with the diagonal action. By [Sch95, Theorem 11.15], $V$ is 2-large. The isotropy groups of $K$ acting on $M$ are just $\{e\}$ and $K$ since the isotropy groups of closed orbits of $G$ acting on $V$ are just $\{e\}$ and $G$. Let $1 \leq k \leq n-2$ and consider the point $x=\left(\left(e_{1}, \ldots, e_{k}, 0, \ldots, 0\right),\left(0, \ldots, 0, e_{1}^{*}, \ldots, e_{k}^{*}\right)\right) \in V \oplus V^{*}$. Here the $e_{i}$ and $e_{i}^{*}$ are the usual basis and dual basis of $\mathbb{C}^{n}$. Then $x \in N$ lies on a closed $G$-orbit with isotropy group $\mathrm{SL}_{n-k}(\mathbb{C})$.

2.3. Symplectic singularities and graded Gorenstein rings. Recall the following.

Definition 2.12 (Beauville, Bea00). An algebraic variety $X$ over $\mathbb{C}$ has a symplectic singularity at $p \in X$ if there is an open neighborhood $U$ of $p$ (classical topology) such that

(i) $U$ is normal,

(ii) the smooth locus $U_{\mathrm{sm}}$ of $U$ admits a holomorphic symplectic (i.e., closed and non-degenerate) 2 -form $\omega$, and

(iii) for any resolution $f: Y \rightarrow U$ of singularities, $\left(\left.f\right|_{f^{-1}\left(U_{\mathrm{sm}}\right)}\right)^{*} \omega$ extends to a holomorphic 2-form on $Y$.

We say that $X$ has symplectic singularities or is a symplectic variety if it has a symplectic singularity at each point.

Note that in condition (iii) of this definition, if there is a resolution $f: Y \rightarrow U$ such that $\left(\left.f\right|_{f^{-1}\left(U_{\mathrm{sm}}\right)}\right)^{*} \omega$ extends to a symplectic form on $Y$, then $Y$ is called a symplectic resolution of $U$. Symplectic varieties need not admit symplectic resolutions. See [Fu06] for a survey of symplectic singularities and resolutions.

As noted in Bea00], the main theorem of [Fle88] implies that condition (iii) of Definition 2.12 is always satisfied if the variety is smooth in codimension 3; that is, we have the following. 
Theorem 2.13 (Flenner, Fle88). Let $X$ be a normal variety such that $X \backslash X_{\mathrm{sm}}$ has codimension at least 4 . Then $X$ has symplectic singularities if and only if $X_{\mathrm{sm}}$ admits a holomorphic symplectic form.

Similarly, using the results of Rei80, Proposition (1.2), Remark (1.3), and Theorem 7], Beauville demonstrates [Bea00, Proposition 1.3] that symplectic varieties are Gorenstein with rational singularities. A converse is proven in Nam01, Theorem 6], yielding the following.

Theorem 2.14 (Namikawa, Nam01]). A normal variety $X$ has symplectic singularities if and only if it is Gorenstein with rational singularities and the smooth locus $X_{\mathrm{sm}}$ admits a holomorphic symplectic form.

Let $R$ be an $\mathbb{N}$-graded algebra of dimension $d$ such that $R_{0}$ is equal to the ground field. Recall [GW78, Definition 2.1.2] that the canonical module of $R$ is defined to be the dual of the $d$ th local cohomology group $\underline{H}_{\mathfrak{m}}^{d}(R)$ where $\mathfrak{m}$ denotes the maximal homogeneous ideal; see Section 4.2 for an explicit description. An algebraic variety $X$ is Cohen-Macaulay if and only if its dualizing complex is a sheaf, corresponding to the canonical module, and is Gorenstein if and only if this sheaf is invertible, see Ish14, Note 3.5.9, Proposition 3.5.12, and Definition 6.2.1] and Sta18, Tag 0A7A, Tag 0DW4, Tag 0DW6. Recall further that $R$ is Gorenstein if and only if it is graded isomorphic to its canonical module, possibly with a shift in the grading, see [Eis95, Section 21.11] GW78, Proposition 2.1.3]. This shift is given by $-(a(R)+d)$ where $a(R)$ is the a-invariant of $R$ [BH93, Definition 3.6.13], GW78, Section 3]. If $a(R)$ is equal to the negative Krull dimension of $R$, then we say that $R$ is graded Gorenstein, also called strongly Gorenstein [DK02, page 103].

By [Sta78, Theorem 4.4], for a Cohen-Macaulay ring $R$, the Gorenstein and graded Gorenstein properties can be determined from the Hilbert series of $R$. Specifically, we have the following.

Theorem 2.15 (Stanley, Sta78). Let $R$ be a graded commutative Noetherian algebra over a field $k=R_{0}$. Suppose that $R$ is a Cohen-Macaulay integral domain with Krull dimension $d$. Then $R$ is Gorenstein if and only if the Hilbert series $\operatorname{Hilb}_{R}(t)$ satisfies

$$
\operatorname{Hilb}_{R}(1 / t)=(-1)^{d} t^{-a} \operatorname{Hilb}_{R}(t)
$$

for an integer $a$, which is then equal to $a(R)$.

If $G$ is finite, then the moment map is trivial so that the complex symplectic quotient is the usual quotient $\left(V \oplus V^{*}\right) / G$. We refer to a quotient of this form as a complex symplectic orbifold; note that we consider orbifolds as varieties with quotient singularities rather than the finer structures of Deligne-Mumford stacks or Lie groupoids. Because $G$ acts as a subgroup of $\operatorname{SL}\left(V \oplus V^{*}\right)$, we have the following consequence of [Bea00, Proposition 2.4] and [Wat74, Theorem 1].

Theorem 2.16 (Beauville Bea00 and Watanabe Wat74). Suppose that $G$ is a finite group and $V$ is a $G$-module. Then the complex symplectic orbifold $\left(V \oplus V^{*}\right) / G$ has symplectic singularities and is graded Gorenstein.

The fact that $\left(V \oplus V^{*}\right) / G$ has rational singularities follows from a theorem of Boutot Bou87, which applies for any reductive $G$. 


\section{Auxiliary Results}

3.1. Normality of the shell. By [HS13, Theorem 2.2(4)], if the $G$-module $V$ is 2large, then the shell $N$ is a normal variety. However, this condition is sufficient but not necessary: there are $G$-modules $V$ that are not 2-large such that $N$ is normal. For example, letting $V \simeq \mathbb{C}^{n}$ denote the defining representation of $\mathrm{SO}_{n}(\mathbb{C})$, the module $\left(V^{\oplus n}, \mathrm{SO}_{n}(\mathbb{C})\right)$ is 2-large while the module $\left(V^{\oplus n}, \mathrm{O}_{n}(\mathbb{C})\right)$ is not. Because the shell coincides for both cases, it is normal [CHS16, page 17]. See also Section 5 below. In fact, using the isomorphism of $\mathbb{C}[N]$ with the symmetric algebra of the Jacobian module described in [HS13, Remark 2.4], assuming $V_{(0)} \neq \emptyset$, the condition that $(V, G)$ is 2-modular is equivalent to the shell $N$ being factorial by Avr81, Proposition 6].

Remark 3.1. If the abelianization $G /[G, G]$ of $G$ is trivial and $(V, G)$ is 3-large, then Bellamy and Schedler have proven that the complex symplectic quotient $N / / G$ is locally factorial [BS18, Theorem 1.2(c)]. This can also be established using the above fact that $N$ is in this case factorial along with Dré04, Theorem 8.4]. In particular, the principal isotropy group is the kernel of the $G$-action on $V \oplus V^{*}$ Sch95, Corollary $7.7(2)]$ so that by taking the quotient, we may assume that $(V, G)$ has TPIG. Take the saturated open set in Dré04, Theorem 8.3] to be the union of the principal orbits. As $N$ is factorial, $\operatorname{Pic}(N)$ is trivial by Ben93, Proposition 3.5.1]. It remains only to show that the units $\mathbb{C}[N]^{*}$ of $\mathbb{C}[N]$ are constant. However, $N$ is a cone and hence stable under the scalar action of $\mathbb{C}^{\times}$. It is verified in the proof of $\left[\right.$ KKV89, Proposition, Section 1.3] that $\mathbb{C}^{\times}$acts on each element $f \in \mathbb{C}[N]^{*}$ as multiplication by a character $\chi$, i.e. $f \circ t=\chi(t) f$ for $t \in \mathbb{C}^{\times}$. Then $f$ must be homogeneous and cannot vanish at the origin, implying that $f$ is constant.

In this section, we give the following characterization of $G$-modules $V$ such that $N$ is normal; compare the similar conditions of [KLS06, Lemma 3.3] as well as the related smoothness criterion in [DH15, Lemma 2.1].

Proposition 3.2. Suppose that $(V, G)$ is 0 -modular, and let $R=\left\{(x, \xi) \in V \oplus V^{*} \mid\right.$ $G_{(x, \xi)}$ is finite\}.

(i) The shell $N$ is a complete intersection, hence $\mathbb{C}\left[V \oplus V^{*}\right] /(\mu)$ is CohenMacaulay.

(ii) The shell $N$ is reduced and irreducible if and only if $V$ is 1-modular.

(iii) If $V$ is 2-modular, then $N$ is normal.

(iv) The set of smooth points $N_{\mathrm{sm}}$ of $N$ is the set of points in $N$ on which $d \mu$ has maximal rank.

(v) The set $R$ is the set of points in $V \oplus V^{*}$ on which $d \mu$ has maximal rank.

(vi) The shell $N$ is normal if and only if $N \backslash(N \cap R)$ has complex codimension at least two in $N$.

Proof. As $(V, G)$ is 0-modular, $(i)$ follows from [Sch95, Proposition 9.4]. In particular, $N$ is equidimensional.

For each $r \geq 0$, let $N_{r}$ denote the closure of $\left(V_{(r)} \times V^{*}\right) \cap N$, and note that each

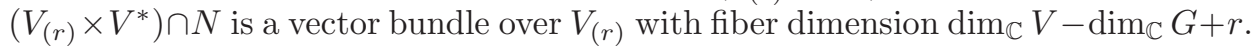
Because $(V, G)$ is 0 -modular, $\operatorname{dim}_{\mathbb{C}} V_{(r)} \leq \operatorname{dim}_{\mathbb{C}} V-r$ for each $r$. Assume $(V, G)$ is not 1-modular, and then there is an $r>0$ such that $\operatorname{dim}_{\mathbb{C}} V_{(r)}=\operatorname{dim}_{\mathbb{C}} V-r$, implying $\operatorname{dim}_{\mathbb{C}} N_{r}=\operatorname{dim}_{\mathbb{C}} N_{0}$. But then $N_{r}$ is not a subset of $N_{0}$, implying that $N$ is not irreducible. Now assume $(V, G)$ is 1 -modular, and then for each $r \geq 1$, 
$\operatorname{dim}_{\mathbb{C}} V_{(r)} \leq \operatorname{dim}_{\mathbb{C}} V-r-1$ so that $\operatorname{dim}_{\mathbb{C}} N_{r}<\operatorname{dim}_{\mathbb{C}} N_{0}$. As $N$ is equidimensional, it must then be that $N_{r} \subset N_{0}$ for each $r \geq 1$. Hence $N$ is equal to the closure of $N_{0} \backslash \bigcup_{r \geq 1} N_{r}$. As $V_{(0)}$ is irreducible, and as $\left(V_{(0)} \times V^{*}\right) \cap N$ is a complex vector bundle over $V_{(0)}$, its closure $N_{0}$ is irreducible. Then $N_{0} \backslash \bigcup_{r \geq 1} N_{r}$ is as well irreducible and smooth, hence reduced, proving (ii). To prove (iii), note that if $(V, G)$ is 2-modular, then the $N_{r}$ have complex codimension at least 2 in $N$. Then $N$ is smooth in codimension 1 and Cohen-Macaulay by $(i)$, hence normal by Serre's criterion Mat89, Theorem 23.8].

As $N$ is a complete intersection, $(i v)$ is simply the Jacobian criterion. Now, for each $A \in \mathfrak{g}$, let $\mu^{A}: V \oplus V^{*} \rightarrow \mathbb{C}$ denote the evaluation of $\mu$ at $A$. As $\mu$ is bilinear, $d \mu^{A}(x, \xi)=0$ if and only if $A(x)=\xi \circ A=0$. Then $(v)$ follows from the fact that $R$ is the set of points where no $d \mu^{A}$ vanishes for $A \in \mathfrak{g} \backslash\{0\}$, and (vi) follows from the fact that $N$ is then smooth in codimension 1 if and only if $N \backslash(N \cap R)$ has codimension at least 2 in $N$.

See [HS13, Remark 2.4] for an alternate proof of (ii) and (iii).

3.2. The $k$-large property for $V^{*}$. Let $G$ be a reductive group and $V$ a $G$ module. The goal of this section is to demonstrate that several properties of $V$ imply the same for the dual module $V^{*}$. We collect these in the following.

Theorem 3.3. Let $G$ be a reductive group and $V$ a $G$-module.

(i) The $G$-module $V$ is stable if and only if $V^{*}$ is stable.

(ii) The $G$-module $V$ has TPIG (respectively FPIG) if and only if $V^{*}$ has TPIG (respectively FPIG).

(iii) The $G$-module $V$ is $k$-modular if and only if $V^{*}$ is $k$-modular.

(iv) The $G$-module $V$ is k-principal if and only if $V^{*}$ is $k$-principal.

(v) The $G$-module $V$ is $k$-large if and only if $V^{*}$ is $k$-large.

Let $\mathrm{Cl}(V)$ denote the set of conjugacy classes $\left(G_{v}\right)$ of isotropy groups of closed orbits $G v$ in $V$. For $(H) \in \mathrm{Cl}(V)$ let $(V / / G)_{(H)}$ denote the corresponding stratum of $V / / G$ and let $V^{(H)}$ denote the inverse image of $(V / / G)_{(H)}$ in $V$. We need the following lemma.

Lemma 3.4. We have $\mathrm{Cl}(V)=\mathrm{Cl}\left(V^{*}\right)$ and for each $(H) \in \mathrm{Cl}(V)$ we have $\operatorname{dim}_{\mathbb{C}}(V / / G)_{(H)}=\operatorname{dim}_{\mathbb{C}}\left(V^{*} / / G\right)_{(H)}$ and $\operatorname{dim}_{\mathbb{C}} V^{(H)}=\operatorname{dim}_{\mathbb{C}}\left(V^{*}\right)^{(H)}$.

Proof. We follow ideas of the proof of [KS01, Proposition E]. First assume that $G$ is connected. Then there is an automorphism $\tau$ of $G$ which interchanges $W$ and $W^{*}$ for every irreducible $G$-module $W$. Specifically, $\tau$ induces an automorphism of the weights of $G$-modules sending the highest weight of $W$ to that of $W^{*}$. Thus we may consider $V^{*}$ as a representation of $G$ on the same space $V$ but with $g \in G$ acting via $\tau(g)$. Then $\mathbb{C}[V]^{G}=\mathbb{C}\left[V^{*}\right]^{G}$ and for each isotropy group $H$, the identification sends $V^{(H)}$ to $\left(V^{*}\right)^{(\tau(H))}$ and sends $(V / / G)_{(H)}$ to $\left(V^{*} / / G\right)_{(\tau(H))}$.

Returning to the general case so that $G$ need not be connected, let $(H) \in$ $\mathrm{Cl}(V)$. Then by Sch80, Lemma 5.5], $V^{H}$ has a Zariski open subset consisting of the intersection of $V^{H}$ with closed orbits with isotropy group $H$. Let $V^{\langle H\rangle}$ denote this intersection. Then the image of $V^{\langle H\rangle}$ in $V / / G$ is $(V / / G)_{(H)}$. Because $H$ is an isotropy group of $V$, the action of $N_{G}(H) / H$ on $V^{H}$ has TPIG, and $\operatorname{dim}_{\mathbb{C}} V^{H} / / N_{G}(H)=\operatorname{dim}_{\mathbb{C}}(V / / H)_{(H)}$. By [KS01, Proposition E(2)], the action of 
$N_{G}(H) / H$ on $\left(V^{H}\right)^{*} \simeq\left(V^{*}\right)^{H}$ has TPIG as well. By Lun75, Corollaire 1], a $G$ orbit through $x$ in $\left(V^{*}\right)^{H}$ is closed if and only if $\left(N_{G}(H) / H\right) x$ is closed. Hence there is a Zariski open subset $U_{H}^{*}$ of $\left(V^{*}\right)^{H}$ consisting of points with closed $G$-orbit. Then there is an $H^{\prime}$ with $\left(H^{\prime}\right) \in \mathrm{Cl}\left(V^{*}\right)$ such that $U_{H}^{*}$ has an open intersection with $\left(V^{*}\right)^{\left\langle H^{\prime}\right\rangle}$ where $H \subset H^{\prime}, \operatorname{dim}_{\mathbb{C}} H^{\prime}=\operatorname{dim}_{\mathbb{C}} H$, and $\left(V^{*}\right)^{H^{\prime}} \subset\left(V^{*}\right)^{H}$. Then as $\left(V^{*}\right)^{H} \simeq\left(V^{H}\right)^{*}$ and similarly for $H^{\prime},\left(V^{*}\right)^{H^{\prime}}=\left(V^{*}\right)^{H}$ and $V^{H}=V^{H^{\prime}}$. Applying the same argument to $\left(H^{\prime}\right) \in \mathrm{Cl}\left(V^{*}\right)$, we see that $H \subset H^{\prime} \subset H^{\prime \prime}$ where $H^{\prime \prime}$ is the isotropy group of closed $G$-orbits intersecting an open subset of $V^{H^{\prime \prime}}=V^{H^{\prime}}=V^{H}$. Hence $H=H^{\prime \prime}=H^{\prime}$ and $\operatorname{dim}_{\mathbb{C}}(V / / G)_{(H)}=\operatorname{dim}_{\mathbb{C}}\left(V^{*} / / G\right)_{(H)}$.

Let $\left(W \oplus \mathbb{C}^{d}\right)$ be the slice representation of $H$ where $W^{H}=0$ and $H$ acts trivially on $\mathbb{C}^{d}$. Then $V \simeq \mathfrak{g} / \mathfrak{h} \oplus W \oplus \mathbb{C}^{d}$ as $H$-module. Letting $e=\operatorname{dim}_{\mathbb{C}}(V / / G)_{(H)}$, the dimension of $V^{(H)}$ is $\operatorname{dim}_{\mathbb{C}} \mathfrak{g} / \mathfrak{h}+e+\operatorname{dim}_{\mathbb{C}} \mathcal{N}(W)$ where $\mathcal{N}(W)$ is the null cone of $W$. For the action of $H$ on $V^{*}$ we get the decomposition $V^{*} \simeq \mathfrak{g} / \mathfrak{h} \oplus W^{*} \oplus \mathbb{C}^{e}$, so it remains only to show that $\operatorname{dim}_{\mathbb{C}} \mathcal{N}(W)=\operatorname{dim}_{\mathbb{C}} \mathcal{N}\left(W^{*}\right)$. But as a set, the null cone of $W$ is determined by the action of $H^{0}$, and the identification of the $H^{0}$-modules $W$ with $W^{*}$ such that $\mathbb{C}[W]^{H^{0}}=\mathbb{C}\left[W^{*}\right]^{H^{0}}$ identifies the null cones. Thus $\operatorname{dim}_{\mathbb{C}} V^{(H)}=\operatorname{dim}_{\mathbb{C}}\left(V^{*}\right)^{(H)}$.

Remark 3.5. Let $V$ be a $G$-module with FPIG. Choose a basis for $\mathfrak{g}$ and let $\mu_{1}, \ldots, \mu_{m}$ denote the component functions of $\mu$ with respect to this basis. It can be shown that in this context, the $k$-large property for $(V, G)$ is equivalent to the existence of homogeneous $f_{1}, \ldots, f_{k} \in \mathbb{C}[V]^{G}$, vanishing on the inverse images in $V$ of the non-principal strata of $V / / G$, such that $f_{1}, \ldots, f_{k}, \mu_{1}, \ldots, \mu_{m}$ is a regular sequence in $\mathbb{C}\left[V \oplus V^{*}\right]$. See [Sch95, 8.2 and Lemma 9.7]. We will need this fact in Proposition 3.20 below.

Proof of Theorem [3.3. We first note that $(i)$ is proven in [KS01, Proposition E], where it is also demonstrated that the principal isotropy groups of $V$ and $V^{*}$ are the same. Then $(i i)$ is an immediate consequence. Since $(V, G)$ is $k$-modular if and only if $\left(V, G^{0}\right)$ is $k$-modular, (iii) is immediate using the automorphism $\tau$ of Lemma 3.4. Statement $(i v)$ follows directly from Lemma 3.4 and, of course, $(v)$ follows from (ii), (iii) and (iv).

3.3. The $k$-large property is generic. In this section, we support the claim that "most" $G$-modules are 3-large by demonstrating the following.

Theorem 3.6. Let $G$ be connected and semisimple and let $k \in \mathbb{N}$. Among $G$ modules $V$ such that $V^{G}=\{0\}$ and every irreducible component of $V$ is a faithful $\mathfrak{g}$-module, there are up to isomorphism only finitely many $V$ that are not $k$-large.

This theorem was established for the case $k=2$ in [Sch95, Corollary 11.6(2)]. Note that for $G$ simple, Theorem 3.6 implies that all but finitely $G$-modules $V$ such that $V^{G}=\{0\}$ are $k$-large.

To begin, let $Y(G)$ denote the set of 1-parameter subgroups of $G$. Let $\lambda \in Y(G)$ and let $V_{\lambda}^{+}$(respectively $V_{\lambda}^{-}$) denote the subspace of $V$ on which $\lambda$ has strictly positive (respectively strictly negative) weights. Similarly let $V_{\lambda}^{0}$ be the subspace of $V$ fixed by $\lambda$. Then by [Gor90, Lemma 6], there is a constant $d \geq 2$, depending only on $G$, such that

$$
\operatorname{dim}_{\mathbb{C}} V_{\lambda}^{-} \geq \frac{\operatorname{dim}_{\mathbb{C}}\left(V_{\lambda}^{+} \oplus V_{\lambda}^{-}\right)}{d}
$$


Then we have the following.

Corollary 3.7. Let $v \in V$ have closed $G$-orbit, let $H=G_{v}$, and assume $\operatorname{dim}_{\mathbb{C}} H>$ 0 . Let $W$ be the slice representation at $v$ and let $\lambda \in Y(H)$. Then

$$
\operatorname{dim}_{\mathbb{C}}\left(W_{\lambda}^{-} \oplus(\mathfrak{g} / \mathfrak{h})_{\lambda}^{-}\right) \geq \frac{\operatorname{dim}_{\mathbb{C}}\left(W_{\lambda}^{+} \oplus W_{\lambda}^{-}\right)}{d} .
$$

Proof. This follows from Equation (3.1) and the fact that $V \simeq \mathfrak{g} / \mathfrak{h} \oplus W$ as $H$ module.

We also have the following bound on the codimension of the null cone $\mathcal{N}(W)$ of $W$. Let $\widehat{W}$ be an $H$-complement of $W^{H}$ so that $W=W^{H} \oplus \widehat{W}$. Here, we use the notation $\operatorname{codim}(A, B)$ for the codimension of $A$ in $B$ to avoid ambiguities.

Lemma 3.8. Suppose that $\operatorname{dim}_{\mathbb{C}} H>0$. Then

$$
\operatorname{codim}_{\mathbb{C}}(\mathcal{N}(\widehat{W}), \widehat{W}) \geq \min _{\lambda \in Y(H)}\left\{\operatorname{dim}_{\mathbb{C}}\left(W_{\lambda}^{-} \oplus \widehat{W}_{\lambda}^{0}\right)\right\}-\operatorname{dim}_{\mathbb{C}} U
$$

where $U$ is a maximal unipotent subgroup of $H$.

Note that $\mathcal{N}(\widehat{W})=\mathcal{N}(W)$.

Proof. Let $T$ be a maximal torus of $H$ and let $Y^{\prime}(T)$ denote the set of $\lambda \in Y(T)$ which are in the positive Weyl chamber. Note that for $\lambda \in Y(H), \widehat{W}_{\lambda}^{ \pm}=W_{\lambda}^{ \pm}$. By the Hilbert-Mumford criterion [PV94, Theorem 5.2], there are finitely many $\lambda \in Y^{\prime}(T)$ such that $\mathcal{N}(\widehat{W})$ is the union of the $H W_{\lambda}^{+}$. As each $W_{\lambda}^{+}$is stabilized by a Borel subgroup $B$ of $H, \operatorname{dim}_{\mathbb{C}} H W_{\lambda}^{+}=\operatorname{dim}_{\mathbb{C}} U W_{\lambda}^{+}$where $U$ is the unipotent subgroup opposite to the unipotent radical of $B$. Then the fact that $\operatorname{codim}_{\mathbb{C}}\left(W_{\lambda}^{+}, \widehat{W}\right)=\operatorname{dim}_{\mathbb{C}}\left(W_{\lambda}^{-} \oplus \widehat{W}_{\lambda}^{0}\right)$ completes the proof.

Using Corollary 3.7, we can rewrite Equation (3.2) in Lemma 3.8 as

$$
\begin{aligned}
\operatorname{codim}_{\mathbb{C}}(\mathcal{N}(\widehat{W}), \widehat{W}) & \geq \min _{\lambda \in Y(H)}\left\{\frac{\operatorname{dim}_{\mathbb{C}}\left(W_{\lambda}^{+} \oplus W_{\lambda}^{-}\right)}{d}+\operatorname{dim}_{\mathbb{C}} \widehat{W}_{\lambda}^{0}-\operatorname{dim}_{\mathbb{C}} U-\operatorname{dim}_{\mathbb{C}}(\mathfrak{g} / \mathfrak{h})\right\} \\
& \geq \frac{\operatorname{dim}_{\mathbb{C}} \widehat{W}}{d}-\operatorname{dim}_{\mathbb{C}} U-\operatorname{dim}_{\mathbb{C}} \mathfrak{g} .
\end{aligned}
$$

Because $V \simeq W^{H} \oplus \widehat{W} \oplus(\mathfrak{g} / \mathfrak{h})$ as $H$-module, we have

$$
\operatorname{dim}_{\mathbb{C}} \widehat{W}=\operatorname{codim}_{\mathbb{C}}\left(W^{H}, V\right)-\operatorname{dim}_{\mathbb{C}}(\mathfrak{g} / \mathfrak{h}) \geq \operatorname{codim}_{\mathbb{C}}\left(V^{H}, V\right)-\operatorname{dim}_{\mathbb{C}}(\mathfrak{g} / \mathfrak{h}) .
$$

By Gor90, Proposition 6], as soon as $\operatorname{dim}_{\mathbb{C}} V \geq \operatorname{dim}_{\mathbb{C}} G$, we have for any $L \leq G$ with nontrivial action on $V$ that

$$
\operatorname{codim}_{\mathbb{C}}\left(V^{L}, V\right) \geq \frac{\sqrt{\operatorname{dim}_{\mathbb{C}} V}}{17} .
$$

Combining these observations with $L=H$ yields the following.

Corollary 3.9. If $\operatorname{dim}_{\mathbb{C}} V \geq \operatorname{dim}_{\mathbb{C}} G$, then

$$
\operatorname{codim}_{\mathbb{C}}(\mathcal{N}(\widehat{W}), \widehat{W}) \geq \frac{\sqrt{\operatorname{dim}_{\mathbb{C}} V}}{17 d}-\operatorname{dim}_{\mathbb{C}} U-2 \operatorname{dim}_{\mathbb{C}} \mathfrak{g} .
$$

With this, we proceed with the proof of the main result of this section. 
Proof of Theorem 3.6. Let $v \in V$ have closed $G$-orbit, let $H=G_{v}$, let $W$ be the slice representation at $v$, and write $W=W^{H} \oplus \widehat{W}$ as above. Recall from Section 3.2 that $(V / / G)_{(H)}$ denotes the stratum of points with isotropy group conjugate to $H$ and $V^{(H)}$ denotes its inverse image in $V$. It follows from Luna's slice theorem Lun73 that the codimension of $V^{(H)}$ in $V$ is equal to the codimension of the null cone $\mathcal{N}(\widehat{W})$ in $\widehat{W}$. If $H$ is finite and nontrivial, then this is just the codimension of $W^{H}$ in $W$, which is bounded below by the codimension of $V^{H}$ in $V$ minus the dimension of $\mathfrak{g}$. Applying Equation (3.3), we have that $\operatorname{codim}_{\mathbb{C}} V^{(H)} \rightarrow \infty$ as $\operatorname{dim}_{\mathbb{C}} V \rightarrow \infty$. If $\operatorname{dim}_{\mathbb{C}} H>0$, then $\operatorname{codim}_{\mathbb{C}}(\mathcal{N}(\widehat{W}), \widehat{W}) \rightarrow \infty$ as $\operatorname{dim}_{\mathbb{C}} V \rightarrow \infty$ by Corollary 3.9 and Equation (3.3).

Now, note that $V \backslash V_{\text {pr }}$ is the union of the $V^{(H)}$ for non-principal isotropy types $(H)$ corresponding to closed orbits. The estimates above imply that $V$ is $k$-principal for $\operatorname{dim}_{\mathbb{C}} V$ sufficiently large. Similarly, for all but finitely many $V$, each $V^{(H)}$ with $H$ infinite has positive codimension. As the complement of the $V^{(H)}$ consists of points with closed orbit and finite isotropy, all but finitely many $V$ have FPIG. Then by the proof of Sch95, Corollary 11.6], replacing the inequality $\kappa_{m}(V) \geq 2$ with $\kappa_{m}(V) \geq k$, there are up to isomorphism only finitely many $V$ which are not $k$-modular. Thus all but finitely many $\mathrm{V}$ are $k$-large.

3.4. The symplectic slice theorem for complex symplectic quotients. In this section, we adapt Luna's étale slice theorem [Lun73] to the context of the Hamiltonian actions considered in this paper. We use this formulation to demonstrate that for 1-large $(V, G)$ such that $N$ is normal and $(N / / G)_{\mathrm{pr}}=(N / / G)_{\mathrm{sm}}$, the symplectic form on $V \oplus V^{*}$ induces a symplectic form on the smooth points of the complex symplectic quotient. This strengthens the criteria given in Bec10. Proposition 2.4]; see also [DH15, Lemma 2.5]. The results of this section are closely related to those of [BLLT17, Section 6]. We give a slightly more explicit description of the shell in the slice representation in order to understand how the slice representation inherits properties such as 1-modular from $(V, G)$, and as well to determine bounds on the codimensions of the non-principal points.

We begin with the following observations regarding the structure of $G$-modules in the presence of invariant bilinear forms.

Lemma 3.10. Let $V$ be a $G$-module which admits non-degenerate $G$-invariant bilinear forms $\omega$ and $\sigma$ such that $\omega$ is skew-symmetric and $\sigma$ is symmetric. Then $V \simeq W \oplus W^{*}$ for some $G$-module $W$, and $W$ and $W^{*}$ are isotropic relative to $\omega$.

Proof. Define the linear map $\tau: V \rightarrow V$ by $\omega\left(v, v^{\prime}\right)=\sigma\left(\tau(v), v^{\prime}\right)$ for $v, v^{\prime} \in V$. In coordinates, if $\Omega$ and $\Sigma$ denote the matrices of $\omega$ and $\sigma$, respectively, then $\tau$ is given by $-\Sigma^{-1} \Omega$. Hence $\tau$ is $G$-equivariant and skew-symmetric. The eigenvalues of $\tau$ are in pairs of the form $\pm \lambda i$ for $\lambda>0$. Thus we can write $V=V_{+} \oplus V_{-}$where $V_{+}$ (respectively $V_{-}$) is the sum of the eigenspaces for positive (respectively negative) multiples of $i$. Then $\omega$ vanishes on $V_{+}$and $V_{-}$and therefore induces a dual pairing of $V_{+}$and $V_{-}$, hence $V \simeq V_{+} \oplus\left(V_{+}\right)^{*}$.

Corollary 3.11. Let $V$ be a $G$-module and let $U$ be a $G$-submodule such that $\widetilde{V}:=V \oplus V^{*}$ contains a submodule of the form $\widetilde{U} \simeq U \oplus U^{*}$. Then there is a $G$-module $W$ and a $G$-submodule $\widetilde{W} \subset \widetilde{V}$ of the form $\widetilde{W} \simeq W \oplus W^{*}$ such that $\widetilde{V}=\widetilde{U} \oplus \widetilde{W}$. 
Proof. We follow an argument from Lun72, Lemma 5]. Let $\omega_{1}$ be a non-degenerate $G$-invariant bilinear skew-symmetric form on $\widetilde{V}$ and let $\omega_{2}$ be a non-degenerate $G$-invariant bilinear skew-symmetric form on $\widetilde{U}$. We extend $\omega_{2}$ by zero on a $G$ complement to $\widetilde{U}$ in $\widetilde{V}$ to consider it as a form on $\widetilde{V}$. Consider the $G$-invariant skew-symmetric forms $\omega_{t}:=t \omega_{1}+(1-t) \omega_{2}$ for $t \in \mathbb{R}$. For most $t$, the form $\omega_{t}$ is non-degenerate on both $\widetilde{V}$ and $\widetilde{U}$. Let $t_{0}$ be such a $t$, and let $\widetilde{W}$ denote the orthogonal complement to $\widetilde{U}$ with respect to $\omega_{t_{0}}$. Then $\omega_{t_{0}}$ is non-degenerate on $\widetilde{W}$ and induces a non-degenerate form on $\widetilde{V} / \widetilde{U} \simeq \widetilde{W}$. The same argument shows that $\widetilde{V} / \widetilde{U}$ admits a non-degenerate $G$-invariant symmetric bilinear form, hence so does $\widetilde{W}$. Now we can apply Lemma 3.10 .

Recall that if $X$ and $Y$ are affine $G$-varieties, a $G$-morphism $\phi: X \rightarrow Y$ is said to be excellent if

(i) $\phi$ is étale,

(ii) the induced morphism $\phi / / G: X / / G \rightarrow Y / / G$ is étale, and

(iii) the morphism $\left(\phi, \pi_{X}\right): X \rightarrow Y \times_{Y / / G} X / / G$ is an isomorphism.

Thinking analytically, $\phi$ is étale if and only if it induces a locally biholomorphic map of the complex analytic varieties associated to $X$ and $Y$. In particular, if $X$ and $Y$ are smooth, $\phi$ is étale if and only if the differential of $\phi$ is an isomorphism at each point of $X$.

Lemma 3.12. Let $X$ and $Y$ be smooth affine $G$-varieties. Let $\omega$ be a symplectic form on $Y$ with respect to which $Y$ is Hamiltonian with moment map $\mu: Y \rightarrow \mathfrak{g}^{*}$. If $\phi: X \rightarrow Y$ is excellent, then $X$ is Hamiltonian with respect to the symplectic form $\phi^{*} \omega$ and moment map $\phi^{*} \mu$.

Proof. For each $x \in X$ and $A \in \mathfrak{g}$, one checks that $d\left(\left(\phi^{*} \mu\right)^{A}\right)(x)=\left(\iota_{A} \phi^{*} \omega\right)(x)$. Hence $\phi^{*} \mu$ is a moment mapping for the symplectic action of $G$ on $X$.

Let $X$ be an affine $G$-variety, and let $x \in X$. Recall that an étale slice at $x$ is a $G_{x}$-invariant locally closed subvariety $S^{\prime}$ of $X$ containing $x$ such that the morphism $G \times \times_{G_{x}} S^{\prime} \rightarrow X$ sending $[g, s] \mapsto g s$ is excellent. Here $G \times \times_{G_{x}} S^{\prime}$ is the quotient of $G \times S^{\prime}$ by the free $G_{x}$-action $h(g, s)=\left(g h^{-1}, h s\right)$ for $h \in G_{x}, g \in G, s \in S^{\prime}$. Then $[g, s]$ denotes the image of $(g, s)$ in $G \times_{G_{x}} S^{\prime}$. By Luna's étale slice theorem [Lun73, there is a slice at each point $x$ such that the orbit $G x$ is closed.

We will now describe the form of an étale slice in the presence of a $G$-invariant symplectic structure at a point in the shell. Let $(V, K)$ be unitary, let $x \in N \subset$ $V \oplus V^{*}$ with $G x$ closed, set $L=G_{x}$, and let $\mathfrak{l} \subset \mathfrak{g}$ denote the Lie algebra of $L$. Let $\perp$ denote orthogonal with respect to the symplectic form. Since $x \in N, E:=T_{x}(G x)$ is isotropic and we have a direct sum decomposition of $L$-modules

$$
V \oplus V^{*} \simeq S \oplus E \oplus E^{*}
$$

where $S \oplus E=E^{\perp}$ and $E^{*} \simeq(\mathfrak{g} / \mathfrak{l})^{*}$ is paired dually with $E \simeq \mathfrak{g} / \mathfrak{l}$ via $\omega$. Then $\omega$ restricts to a symplectic form $\omega_{S}$ on $S$. We call $(S, L)$ the symplectic slice representation at $x$. We will show that, up to an excellent morphism, $N$ near $x$ is isomorphic to $G \times_{L} N_{S}$ at $[e, 0]$ where $N_{S}$ is the zero set of the moment map $\mu_{S}: S \rightarrow \mathfrak{l}^{*}$.

Proposition 3.13. Let $V$ be a $G$-module and let $\omega$ denote the standard symplectic form on $V \oplus V^{*}$. Let $x=(v, \xi) \in N$ such that $G x$ is closed with isotropy group $L$, let $(S, L)$ denote the symplectic slice representation at $x$ and let $\omega_{S}$ denote the 
restriction of $\omega$ to $S$. Then $S=W \oplus W^{*}$ where $W$ is an $L$-module and $W$ and $W^{*}$ are isotropic subspaces relative to $\omega_{S}$.

Proof. By Corollary 3.11, $S \simeq W \oplus W^{*}$ where $W$ is an $L$-module. Since $\omega_{S}$ is non-degenerate, $W$ and $W^{*}$ can be chosen to be isotropic by Lemma 3.10 .

Because $\omega$ is $G$-invariant, as an $L$-module, $V \oplus V^{*}$ is the orthogonal symplectic direct sum $\left(E \oplus E^{*}\right) \oplus\left(W \oplus W^{*}\right)$. For the $G$-action on $V \oplus V^{*}$, the slice representation $S^{\prime}$ at $x$ is $E^{*} \oplus S$. We have the canonical mapping

$$
\phi: G \times_{L}\left(E^{*} \oplus S\right) \rightarrow V \oplus V^{*}, \quad\left[g, e^{*}+s\right] \mapsto g\left(x+e^{*}+s\right) .
$$

By [Lun73, Lemme Fondamental] there is a $G$-saturated neighborhood $U^{\prime}$ of $x \in$ $V \oplus V^{*}$ and an $L$-saturated neighborhood $U$ of $0 \in E^{*} \oplus S$ such that $\phi: G \times{ }_{L} U \rightarrow U^{\prime}$ is excellent. By Lemma 3.12 $G \times{ }_{L} U$ is Hamiltonian with symplectic form $\phi^{*} \omega$ and moment mapping $\phi^{*} \mu$.

Lemma 3.14. There are L-saturated neighborhoods $Q^{\prime}$ of $x \in U$ and $Q$ of $0 \in$ $E^{*} \oplus S$ and an L-equivariant excellent morphism $\widetilde{\psi}: Q \rightarrow Q^{\prime}$ such that $\widetilde{\psi}^{*} \phi^{*} \mu$ has zero set $\left(\{0\} \times N_{S}\right) \cap Q$.

Proof. We make a change of coordinates on $E^{*}+S$. Let $A_{1}, \ldots, A_{m}$ be elements of $\mathfrak{g}$ such that the $A_{i}+\mathfrak{l}$ form a basis of $E \simeq \mathfrak{g} / \mathfrak{l}$. Then the functions $f_{i}=\mu^{A_{i}}$ have linearly independent differentials at $x$ and span the dual of $E^{*}$. Let $h_{1}, \ldots, h_{2 k}$ be a basis of $S^{*}$. Then the $f_{i}$ and $h_{j}$ are a local coordinate system near $x \in x+E^{*}+S$, and the corresponding change of coordinates is equivariant relative to the action of $L$. By Lun73, Lemme Fondamental] we have an excellent mapping $\widetilde{\psi}$ of a neighborhood $Q$ of $0 \in E^{*} \times S$ to a neighborhood $Q^{\prime}$ of $x$ in $x+E^{*}+S$, which we identify with $E^{*} \oplus S$, such that the pull-backs of the $f_{i}$ form a linear coordinate system on $E^{*}$ and the pull-back of each $h_{i}$ is $h_{i}$. By shrinking if necessary, we may assume $Q^{\prime} \subset U$. Evidently, the zero set of the $f_{i}$ is $\{0\} \times S$. Let $A \in \mathfrak{l}$. Then the zero set of $\widetilde{\psi}^{*} \phi^{*} \mu^{A}$ on $\{0\} \times S$ is just the zero set of $\mu^{A}$ on $x+S \subset V \oplus V^{*}$. This establishes the lemma.

Note that $\widetilde{\psi}$ induces an excellent mapping $\psi$ of $G \times{ }_{L} Q \rightarrow G \times{ }_{L} Q^{\prime} \subset G \times{ }_{L} U$. Finally, we have the following symplectic slice theorem.

Theorem 3.15. Let $x$ be a closed orbit in $N$ with isotropy group L. Let $(S, L)$ denote the symplectic slice representation at $x$ with its induced Hamiltonian structure as above. Set $E:=T_{x}(G x)$. Then there is an L-saturated neighborhood $Q$ of $0 \in E^{*} \oplus S$ and an excellent morphism $\rho: G \times{ }_{L} Q \rightarrow V \oplus V^{*}$ such that $\rho([e, 0])=x$, and if $G \times{ }_{L} Q$ is given the induced Hamiltonian structure via $\rho$, then the shell of $G \times{ }_{L} Q$ is $G \times \times_{L}\left(Q \cap\left(\{0\} \times N_{S}\right)\right)$. The induced mapping

$$
\tilde{\rho}: G \times_{L}\left(Q \cap\left(\{0\} \times N_{S}\right)\right) \rightarrow N
$$

is excellent.

Proof. It follows from Lemma 3.14 that $\rho=\phi \circ \psi$ has the desired properties. In particular, as the shell of $G \times{ }_{L} Q$ is $G$-invariant, it is determined by its intersection with $Q$, which is given by $\left(\{0\} \times N_{S}\right) \cap Q$, and $\rho^{-1}(N)=G \times{ }_{L}\left(\widetilde{\psi}^{*} \phi^{*} \mu^{-1}(0)\right)$. Since $\rho$ is excellent, $\rho^{-1}(N) \rightarrow N$ is excellent by PV94, Section 6.2], showing that $\widetilde{\rho}$ is excellent. 
We now consider the properties of $(V, G)$ that are inherited by $(W, L)$ for a symplectic slice representation $W \oplus W^{*}$ corresponding to the isotropy group $L$.

Corollary 3.16. Let $(P)$ be any of the following properties: reduced, smooth, or normal. Then $N_{S}$ has $(P)$ at 0 if and only if $N$ has $(P)$ at $x$. Similarly, $N_{S} / / L$ has $(P)$ at the image of 0 if and only if $N / / G$ has $(P)$ at the image of $x$. The complex codimension of $(N / / G)_{(L)}$ in $N / / G$ is the same as the complex codimension of $\left(N_{S} / / L\right)_{(L)}$ in $N_{S} / / L$, and the complex codimension of $N^{(L)}$ in $N$ is the same as the complex codimension of $N_{S}^{(L)}$ in $N_{S}$.

Proof. Note that the restriction of an excellent mapping to the preimage of a closed invariant set is excellent, see [PV94, Section 6.2]. For étale mappings $\sigma: X \rightarrow Y$, a subvariety $Z$ of $Y$ has property $(P)$ at $y$ if and only if $\sigma^{-1}(Z)$ has property $(P)$ at a point (equivalently, every point) of $\sigma^{-1}(y)$, see SGA1, Corollaire 9.2, Proposition 9.2 , and Corollaire 9.10]. Since $\widetilde{\rho}$ is étale, and since $\widetilde{\rho}$ induces an étale mapping on the quotients, the claims follow.

In particular, note that the shell $N$ is a cone and hence connected. By Corollary 3.16, if $N$ is normal, then $N_{S}$ is normal, implying by Proposition 3.2 (ii) that $(W, L)$ is 1-modular.

Lemma 3.17. Suppose that $(V, G)$ is 1-modular with FPIG. Then the principal isotropy groups of $N$ are finite and $(N / / G)_{\mathrm{pr}} \subset(N / / G)_{\mathrm{sm}}$.

Proof. Since $V$ is 1-modular, $N$ is irreducible by Proposition 3.2 $(i i)$. As $(V, G)$ has FPIG and $N$ contains $V \times\{0\}, N$ has FPIG as well. Let $G x \subset N$ be a principal orbit. Since $G_{x}$ is finite, Proposition $3.2(i v)$ and $(v)$ show that $x$ is a smooth point of $N$. Hence the slice representation at $x$ is trivial, and hence $G x$ is a smooth point of $N / / G$. Thus $(N / / G)_{\mathrm{pr}} \subset(N / / G)_{\mathrm{sm}}$.

Now, suppose that $(V, G)$ is 1-large, the corresponding shell $N$ is normal, and $(N / / G)_{\mathrm{sm}}=(N / / G)_{\mathrm{pr}}$, i.e. every smooth point of $N / / G$ corresponds to a principal orbit. Let $G x \subset N$ be a principal orbit. Then the symplectic form $\omega_{S}$ given by Proposition 3.13 induces a symplectic form on a neighborhood of the orbit $G x$ in $N_{\mathrm{pr}} / / G=(N / / G)_{\mathrm{sm}}$. Recall that $\omega_{S}$ is given by restriction of the standard symplectic form $\omega$ on $V \oplus V^{*}$. Hence, $\omega_{S}$ can be recovered from the Poisson bracket restricted to $S$ via $\omega\left(X_{f}, X_{g}\right)=\{f, g\}$ where $X_{f}$ and $X_{g}$ denote the Hamiltonian vector fields corresponding to functions $f$ and $g$. In particular, as the Hamiltonian vector fields of the coordinate functions of $S$ span the tangent space to $S, \omega_{S}$ is determined by the brackets of the coordinate functions, see Equation (2.3). For $f, g \in \mathbb{C}\left[V \oplus V^{*}\right]^{G}$ representing elements of $\mathbb{C}[N]^{G}$, the bracket $\left\{f+(\mu)^{G}, g+(\mu)^{G}\right\}$ in $\mathbb{C}[N]^{G}$ is defined by $\{f, g\}+(\mu)^{G}$, see Definition 2.5. so that the symplectic form on the image of $S$ in $(N / / G)_{\mathrm{sm}}$ is that induced by the Poisson bracket on $\mathbb{C}[N]^{G}$. Hence we have the following.

Corollary 3.18. Suppose that $(V, G)$ is 1-large, the corresponding shell $N$ is normal, and $(N / / G)_{\mathrm{sm}}=(N / / G)_{\mathrm{pr}}$, and let $\omega$ denote the standard symplectic form on $V \oplus V^{*}$. Then the Poisson bracket on the complex symplectic quotient $\mathbb{C}[N]^{G}$ induces a regular symplectic form on the smooth locus $(N / / G)_{\mathrm{sm}}$ of the complex symplectic quotient $N / / G$ that agrees with the restriction of the standard symplectic form $\omega$ on $V \oplus V^{*}$ to symplectic slices at points with principal isotropy. 
3.5. The condition $(N / / G)_{\mathrm{sm}}=(N / / G)_{\mathrm{pr}}$. In this section, we establish conditions under which the hypothesis $(N / / G)_{\mathrm{sm}}=(N / / G)_{\mathrm{pr}}$ of Corollary 3.18 is true.

First note that by Theorem 3.15 the hypothesis $(N / / G)_{\mathrm{sm}}=(N / / G)_{\mathrm{pr}}$ is equivalent to $N_{S} / / L$ being singular at the origin for every $(W, L)$ corresponding to a symplectic slice $W \oplus W^{*}$ at a point $x \in N$ with closed orbit and non-principal isotropy group $L$. By [HSS15, Lemma 2.3], $N_{S} / / L$ is singular if $(W, L)$ is 1-large so that $(N / / G)_{\mathrm{sm}}=(N / / G)_{\mathrm{pr}}$ holds whenever each of the $(W, L)$ can be chosen to be 1-large.

We have the following, which allows us to reduce without loss of generality to the case of connected groups.

Proposition 3.19. Let $G$ be reductive and let $V$ be a $G$-module such that the shell $N$ is normal and has FPIG. If $N / / G$ is smooth, then $N / / G^{0}$ is smooth.

Proof. Let $x=(v, \xi) \in N$ have image in $\left(N / / G^{0}\right)_{\mathrm{pr}}$. Then $\left(G^{0}\right)_{x}$ is finite and acts trivially on the symplectic slice representation $W \oplus W^{*}$ at $x$. Now, $G_{x}$ also acts on $W \oplus W^{*}$ and contains no pseudoreflections so that as $\left(W \oplus W^{*}\right) / G_{x}$ is smooth, by the Chevalley-Shephard-Todd theorem [ST54, Che55, $G_{x}$ must act trivially on $W \oplus W^{*}$. Therefore, $G / G^{0}$ has a normal subgroup $H$ which acts trivially on $\left(N / / G^{0}\right)_{\mathrm{pr}}$, and hence on $N / / G^{0}$, and the quotient group $F=\left(G / G^{0}\right) / H$ acts freely on $\left(N / / G^{0}\right)_{\text {pr }}$. We claim that $F$ is trivial.

Let $U$ be the open subset of $\mathbb{C}^{2 d}=N / / G$ given by the image of $\left(N / / G^{0}\right)_{\mathrm{pr}}$. Then $\left(N / / G^{0}\right)_{\text {pr }}$ is a covering space of $U$ with covering group $F$. Because the strata of $N / / G^{0}$ have even dimension, the complement of $U$ has complex codimension at least 2 in $N / / G$. By HSS15, Lemma 2.4], $U$ is simply connected so that $F$ is trivial. Hence $N / / G^{0}$ is smooth.

We now prove that $(N / / G)_{\mathrm{sm}}=(N / / G)_{\mathrm{pr}}$ when $(V, G)$ is 3-large, see Theorem 3.22 below. We begin with the following.

Proposition 3.20. If $(V, G)$ is 3-large, then for any symplectic slice representation $(S, L)$ at $x \in N$, the codimension of $N_{S} \backslash\left(N_{S}\right)_{\mathrm{pr}}$ in $N_{S}$ is at least 3 .

Proof. As $V$ is 3-principal, there are $f_{1}, f_{2}, f_{3} \in \mathbb{C}[V]^{G}$ which vanish on $V \backslash V_{\text {pr }}$ and form a regular sequence in $\mathbb{C}[V]$. As $V$ is 3-large, the $f_{i}$ and the coordinates $\mu_{1}, \ldots, \mu_{m}$ of $\mu$ with respect to a basis for $\mathfrak{g}$ form a regular sequence in $\mathbb{C}\left[V \oplus V^{*}\right]$, see Remark 3.5. Thus the zero set $N^{\prime}$ of the $f_{i}$ has codimension 3 in $N$. Let $x=(v, \xi) \in N \backslash N^{\prime}$. Then one of the $f_{i}(v)$ is not zero, which implies that $v \in V_{\mathrm{pr}}$. Hence $x$ is in $N_{\mathrm{pr}}$. In other words, $N \backslash N_{\mathrm{pr}} \subset N^{\prime}$ where $N^{\prime}$ has codimension 3 in $N$. By Corollary 3.16, it follows that for any symplectic slice representation $(S, L)$, $N_{S}^{(L)}$ has codimension at least 3 in $N_{S}$.

We now follow an argument of Sta]. Choose an $L$-complement $\widehat{W}$ of $W^{L}$ so that $W=W^{L} \oplus \widehat{W}$ and let $\widehat{S}=\widehat{W} \oplus \widehat{W}^{*}$ so that $S=S^{L} \oplus \widehat{S}$. Then $N_{S}=S^{L} \times N_{\widehat{S}}$ and $N_{S} / / L=S^{L} \times N_{\widehat{S}} / / L$. Let $X$ be the image of $N_{\widehat{S}} \backslash\{0\}$ in $\mathbb{P}:=\mathbb{P}\left(\widehat{W} \oplus \widehat{W}^{*}\right)$ and let $Z$ be the image of the nonzero points in $N_{\widehat{S}} \backslash\left(N_{\widehat{S}}\right)_{\mathrm{pr}}$. Note that $X$ is a complete intersection and that $X \backslash Z$ is smooth.

Lemma 3.21. The inclusion $X \backslash Z \rightarrow X$ induces an isomorphism $\pi_{1}(X \backslash Z) \rightarrow$ $\pi_{1}(X)$ and a surjection $\pi_{2}(X \backslash Z) \rightarrow \pi_{2}(X)$. 
Proof. Choose a generic linear section $H$ of $\mathbb{P}$ which has codimension $\operatorname{dim}_{\mathbb{C}} X-2$ and does not intersect $Z$. Then GM88, Theorem 1.2] (with $\hat{n}=2$ ) implies that the maps $\pi_{i}(H \cap X) \rightarrow \pi_{i}(X)$ and

$$
\pi_{i}(H \cap(X \backslash Z))=\pi_{i}(H \cap X) \rightarrow \pi_{i}(X \backslash Z) .
$$

are isomorphisms for $i=1$ and surjections for $i=2$.

Theorem 3.22. Suppose $(V, G)$ is 3-large. Then for any symplectic slice representation $\left(W \oplus W^{*}, L\right)$ such that $L$ is not principal, $N_{\widehat{S}} / / L$ is not smooth. Hence, $(N / / G)_{\mathrm{pr}}=(N / / G)_{\mathrm{sm}}$.

Proof. Note that if $(V, G)$ is 2-principal, then the principal isotropy group is the kernel of the homomorphism $G \rightarrow \mathrm{GL}(V)$ by Sch95, Corollary 7.7(2)]. Hence, replacing $G$ with its image in $\operatorname{GL}(V)$, we may assume $V$ has TPIG. As the real shell $M \subset N$ is the Kempf-Ness set and hence intersects each closed orbit in $V$ Sch89, Corollary 4.7], it follows that $N$ contains closed orbits with trivial isotropy and hence has TPIG as a $G$-variety.

If $N_{\widehat{S}} / / L$ is smooth, then the same holds for any of the corresponding symplectic slice representations. Thus it is enough to consider the case that $N_{\widehat{S}} / / L$ is smooth for $\left(W \oplus W^{*}, L\right)$ subprincipal, i.e., the only proper symplectic slice representations in $W \oplus W^{*}$ are principal. Since $N_{\widehat{S}} / / L$ is smooth and a cone, it is isomorphic to affine space of dimension $2 d$ for some $d \geq 1$. The non-principal orbits in $N_{\widehat{S}}$ are just the null cone $\mathcal{N}$ so that the image of the principal orbits in $N_{\widehat{S}} / / L \simeq \mathbb{C}^{2 d}$ is just the complement of the origin. Therefore, $\pi_{i}\left(\left(N_{\widehat{S}} / / L\right)_{\mathrm{pr}}\right)=\pi_{i}\left(\mathbb{C}^{2 d} \backslash\{0\}\right)=0$ for $i=1,2$. Since the action of $L$ on $N_{\widehat{S}}$ has TPIG, we have a fiber bundle $L \rightarrow\left(N_{\widehat{S}}\right)_{\mathrm{pr}} \rightarrow \mathbb{C}^{2 d} \backslash\{0\}$. Taking the exact homotopy sequence, we obtain

$$
\underbrace{\pi_{2}(L)}_{=0} \rightarrow \pi_{2}\left(\left(N_{\widehat{S}}\right)_{\mathrm{pr}}\right) \rightarrow \underbrace{\pi_{2}\left(\mathbb{C}^{2 d} \backslash\{0\}\right)}_{=0} \rightarrow \pi_{1}(L) \rightarrow \pi_{1}\left(\left(N_{\widehat{S}}\right)_{\mathrm{pr}}\right) \rightarrow \underbrace{\pi_{1}\left(\mathbb{C}^{2 d} \backslash\{0\}\right)}_{=0} .
$$

Thus $\pi_{1}(L) \simeq \pi_{1}\left(\left(N_{\widehat{S}}\right)_{\mathrm{pr}}\right)$ and $\pi_{2}\left(\left(N_{\widehat{S}}\right)_{\mathrm{pr}}\right)=0$.

Recall that $\mathbb{P}=\mathbb{P}^{2 \operatorname{dim}_{\mathbb{C}} \widehat{W}-1}$. By [FL81, Corollary 9.7], the relative homotopy groups $\pi_{i}(\mathbb{P}, X)$ vanish for $i \leq 2 \operatorname{dim}_{\mathbb{C}} X-\operatorname{dim}_{\mathbb{C}} \mathbb{P}+1=2 d$, as $\operatorname{dim}_{\mathbb{C}} N_{\widehat{S}}=$ $2 \operatorname{dim}_{\mathbb{C}} \widehat{W}-\operatorname{dim}_{\mathbb{C}} L$. It follows that $\pi_{2}(X)=\mathbb{Z}$ and $\pi_{1}(X)=0$. We have a diagram of fibrations

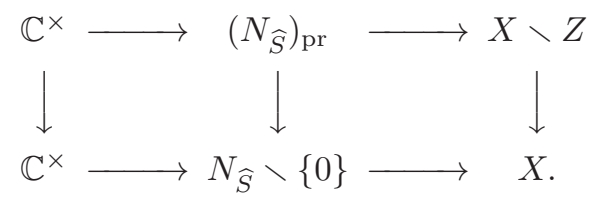

A diagram chase and Lemma 3.21 show that $\pi_{1}\left(\left(N_{\widehat{S}}\right)_{\mathrm{pr}}\right) \simeq \pi_{1}\left(N_{\widehat{S}} \backslash\{0\}\right)$ and that $0=\pi_{2}\left(\left(N_{\widehat{S}}\right)_{\text {pr }}\right)$ maps onto $\pi_{2}\left(N_{\widehat{S}} \backslash\{0\}\right)$, which then must vanish. From the fibration $\mathbb{C}^{\times} \rightarrow N_{\widehat{S}} \backslash\{0\} \rightarrow X$, we then have an exact sequence

$$
0 \rightarrow \underbrace{\pi_{2}(X)}_{=\mathbb{Z}} \rightarrow \underbrace{\pi_{1}\left(\mathbb{C}^{\times}\right)}_{=\mathbb{Z}} \rightarrow \pi_{1}\left(N_{\widehat{S}} \backslash\{0\}\right) \rightarrow \underbrace{\pi_{1}(X)}_{=0} .
$$

Hence $\pi_{1}\left(N_{\widehat{S}} \backslash\{0\}\right)$ is finite. Thus $\pi_{1}\left(\left(N_{\widehat{S}}\right)_{\mathrm{pr}}\right)$ and $\pi_{1}(L)$ are finite. This implies that $L$ is semisimple.

Now, as $N$ is normal, $(W, L)$ is 1-modular by Corollary 3.16. Then by [LV73, Corollaire 1], as $W_{(0)}$ is open and consists of points with finite, hence reductive, 
isotropy, $(W, L)$ must be stable. This implies that $(W, L)$ is 1-large. By HSS15. Lemma 2.3], it follows that $N_{\widehat{S}} / / L$ is not smooth, a contradiction.

\section{Proof of Theorem 1.1}

We are now ready to prove the first of our main results. We divide the proof of Theorem 1.1 into two pieces. In Section 4.1, we use the results above to establish that $N / / G$ has symplectic singularities if $(V, G)$ is 3-large, or more generally is 2large and satisfies $(N / / G)_{\mathrm{pr}}=(N / / G)_{\mathrm{sm}}$. In Section 4.2, assuming that $(V, G)$ is 1-large, $N$ is normal, and $(N / / G)_{\mathrm{pr}}=(N / / G)_{\mathrm{sm}}$, we show that $\mathbb{C}[N]^{G}$ is graded Gorenstein if it is Gorenstein.

4.1. Symplectic singularities of $N / / G$. To establish that symplectic quotients that satisfy the hypotheses of Theorem 1.1 have symplectic singularities, we first demonstrate the following.

Lemma 4.1. Let $L$ be a reductive subgroup of $G$ and $G v$ an orbit in $V$ where $v \in V^{L}$. Then the complex codimension of $(G v)^{L}$ in $G v$ is at least

$$
\operatorname{dim}_{\mathbb{C}} G-\operatorname{dim}_{\mathbb{C}} N_{G}(L)-\operatorname{dim}_{\mathbb{C}} G_{v}+\operatorname{dim}_{\mathbb{C}} L .
$$

Proof. Identify $G v$ with $G / G_{v}$. Then the tangent space $T_{e G_{v}}\left(G / G_{v}\right)$ at the coset $e G_{v}$ of the identity is given by $\mathfrak{g} / \mathfrak{g}_{v}$, and because $L$ is reductive, $T_{e G_{v}}\left(G / G_{v}\right)^{L}=$ $\mathfrak{g}^{L} /\left(\mathfrak{g}_{v}\right)^{L}$. As $\mathfrak{g}^{L}=\operatorname{Lie}\left(C_{G}(L)\right)$, the $C_{G}(L)^{0}$-orbit through $e G_{v}$ is open in $\left(G / G_{v}\right)^{L}$. Equivalently, the orbit of $C_{G}(L)^{0}$ in $(G v)^{L}$ is open at $v$, hence open at any point in $(G v)^{L}$. It follows that $C_{G}(L)^{0}$ acts transitively on each connected component of $(G v)^{L}$. Hence the complex codimension of $(G v)^{L}$ in $G v$ is

$$
\begin{aligned}
\operatorname{dim}_{\mathbb{C}} G- & \operatorname{dim}_{\mathbb{C}} G_{v}-\left(\operatorname{dim}_{\mathbb{C}} C_{G}(L)-\operatorname{dim}_{\mathbb{C}} C_{G_{v}}(L)\right) \\
& \geq \operatorname{dim}_{\mathbb{C}} G-\operatorname{dim}_{\mathbb{C}} G_{v}-\operatorname{dim}_{\mathbb{C}} C_{G}(L)+\operatorname{dim}_{\mathbb{C}} C(L)-\operatorname{dim}_{\mathbb{C}} L+\operatorname{dim}_{\mathbb{C}} L \\
& =\operatorname{dim}_{\mathbb{C}} G-\operatorname{dim}_{\mathbb{C}} G_{v}-\operatorname{dim}_{\mathbb{C}} N_{G}(L)+\operatorname{dim}_{\mathbb{C}} L
\end{aligned}
$$

where we have used the fact that $N_{G}(L) / L=(G / L)^{L}$ has complex dimension $\operatorname{dim}_{\mathbb{C}} C_{G}(L)-\operatorname{dim}_{\mathbb{C}} C(L)$.

Lemma 4.2. Suppose that $k \geq 1$ and that $(V, G)$ is $k$-modular, and let $L$ be a reductive subgroup of $G$. Then for each $p>0$,

$$
\operatorname{codim}_{\mathbb{C}}\left(V^{L} \cap V_{(p)}\right) \geq \operatorname{dim}_{\mathbb{C}} G-\operatorname{dim}_{\mathbb{C}} N_{G}(L)+\operatorname{dim}_{\mathbb{C}} L+k .
$$

Proof. Let $v \in V^{L}$ and let $p=\operatorname{dim}_{\mathbb{C}} G_{v}>0$. Then as $(V, G)$ is $k$-modular, $\operatorname{codim}_{\mathbb{C}} V_{(p)} \geq p+k$. Hence by Lemma 4.1 we see that the complex codimension of $V^{L} \cap V_{(p)}$ in $V$ is at least

$$
p+k+\operatorname{dim}_{\mathbb{C}} G-p-\operatorname{dim}_{\mathbb{C}} N_{G}(L)+\operatorname{dim}_{\mathbb{C}} L=\operatorname{dim}_{\mathbb{C}} G-\operatorname{dim}_{\mathbb{C}} N_{G}(L)+\operatorname{dim}_{\mathbb{C}} L+k .
$$

Lemma 4.3. Suppose that $k \geq 1,(V, G)$ is $k$-large with $T P I G$, and $L$ is a nontrivial finite subgroup of $G$. Then

$$
\operatorname{codim}_{\mathbb{C}} V^{L} \geq \operatorname{dim}_{\mathbb{C}} G-\operatorname{dim}_{\mathbb{C}} N_{G}(L)+k .
$$

Proof. For each $p>0, \operatorname{codim}_{\mathbb{C}}\left(V^{L} \cap V_{(p)}\right) \geq \operatorname{dim}_{\mathbb{C}} G-\operatorname{dim}_{\mathbb{C}} N_{G}(L)+k$ by Lemma 4.2. If $v \in V^{L} \cap V_{(0)}$, then $G v$ lies in $V \backslash V_{\mathrm{pr}}$ which has complex codimension at least $k$ in $V$. Applying Lemma 4.1 completes the proof.

We now can prove the following. 
Theorem 4.4. Let $k \geq 1$ and let $(V, K)$ be unitary where $(V, G)$ is $k$-large, the corresponding shell $N$ is normal, and $(N / / G)_{\mathrm{pr}}=(N / / G)_{\mathrm{sm}}$. Then $N / / G \backslash(N / / G)_{\mathrm{sm}}$ has complex codimension at least $2 k$ in $N / / G$.

Proof. Since the isotropy type strata of $N / / G$ are even dimensional, the case $k=1$ is trivial and we may assume that $k \geq 2$. Then as in the proof of Theorem 3.22 we may assume that $N$ has TPIG as a $G$-variety.

Let $n=\operatorname{dim}_{\mathbb{C}} V$. Let $L$ be a non-principal isotropy group for the action of $G$ on $N$ and let $(v, \xi) \in N$ have closed orbit and isotropy group $L$. Let $S=W \oplus W^{*}$ be the corresponding symplectic slice. By Corollary 3.16, the complex codimension of $(N / / G)_{(L)}$ in $N / / G$ is the same as the complex codimension of $\left(N_{S} / / L\right)_{(L)}$ in $N_{S} / / L$. As $N$ has TPIG as a $G$-variety so that $N_{S}$ has TPIG as an $L$-variety, $\operatorname{dim}_{\mathbb{C}} N_{S} / / L=\operatorname{dim}_{\mathbb{C}} S-2 \operatorname{dim}_{\mathbb{C}} L$.

As an $L$-module,

$$
V \oplus V^{*}=S \oplus \mathfrak{g} / \mathfrak{l} \oplus(\mathfrak{g} / \mathfrak{l})^{*}
$$

Write $S=S^{L} \oplus \widehat{S}$. Then the complex codimension of $\left(N_{S} / / L\right)_{(L)}$ in $N_{S} / / L$ is $\operatorname{dim}_{\mathbb{C}} \widehat{S}-2 \operatorname{dim}_{\mathbb{C}} L$. From the decomposition above,

$$
\operatorname{dim}_{\mathbb{C}} S^{L}=\operatorname{dim}_{\mathbb{C}}\left(V \oplus V^{*}\right)^{L}-2 \operatorname{dim}_{\mathbb{C}} \mathfrak{g}^{L}+2 \operatorname{dim}_{\mathbb{C}} \mathfrak{l}^{L},
$$

so that as $\operatorname{dim}_{\mathbb{C}} S=2 n-2\left(\operatorname{dim}_{\mathbb{C}} G-\operatorname{dim}_{\mathbb{C}} L\right)$, we have

$\operatorname{dim}_{\mathbb{C}} \widehat{S}=2 n-\operatorname{dim}_{\mathbb{C}}\left(V \oplus V^{*}\right)^{L}-2 \operatorname{dim}_{\mathbb{C}} G+2\left(\operatorname{dim}_{\mathbb{C}} C_{G}(L)+\operatorname{dim}_{\mathbb{C}} L-\operatorname{dim}_{\mathbb{C}} C(L)\right)$. Using the fact that $\operatorname{dim}_{\mathbb{C}} N_{G}(L)=\operatorname{dim}_{\mathbb{C}} C_{G}(L)+\operatorname{dim}_{\mathbb{C}} L-\operatorname{dim}_{\mathbb{C}} C(L)$, see the proof of Lemma 4.1, this gives

$$
\operatorname{dim}_{\mathbb{C}} \widehat{S}=2 n-\operatorname{dim}_{\mathbb{C}}\left(V \oplus V^{*}\right)^{L}-2 \operatorname{dim}_{\mathbb{C}} G+2 \operatorname{dim}_{\mathbb{C}} N_{G}(L) .
$$

Then as $\operatorname{dim}_{\mathbb{C}}\left(V \oplus V^{*}\right)^{L}=2 \operatorname{dim}_{\mathbb{C}} V^{L}$, applying Lemmas4.2 and 4.3 yields $\operatorname{dim}_{\mathbb{C}} \widehat{S} \geq$ $2 \operatorname{dim}_{\mathbb{C}} L+2 k$, and the complex codimension of $\left(N_{S} / / L\right)_{(L)}$ in $N_{S} / / L$ is at least $2 k$.

Now, suppose that $(V, G)$ is 2-large and $(N / / G)_{\mathrm{pr}}=(N / / G)_{\mathrm{sm}}$; by Theorem 3.22 this is the case if $(V, G)$ is 3-large. As $(V, G)$ is 2-modular, $N$ is normal, implying that $N / / G$ is normal. The smooth points of $N / / G$ inherit a symplectic 2-form from that of $V$ by Corollary 3.18 . Then combining Theorem 4.4 and Theorem 2.13, we have the following.

Corollary 4.5. Suppose that $(V, G)$ is 2-large and $(N / / G)_{\mathrm{pr}}=(N / / G)_{\mathrm{sm}}$. Then the complex symplectic quotient $N / / G$ has symplectic singularities.

That $N / / G$ is Gorenstein with rational singularities then follows from Theorem 2.14. To complete the proof of Theorem 1.1, then, we need only demonstrate that $N / / G$ is graded Gorenstein; this will be shown in the next section.

4.2. Graded Gorenstein symplectic quotients. The goal of this section is to prove the following.

Theorem 4.6. Assume that $(V, G)$ is 1-large, the corresponding shell $N$ is normal, and $(N / / G)_{\mathrm{pr}}=(N / / G)_{\mathrm{sm}}$. If $N / / G$ is Gorenstein, then it is graded Gorenstein.

Clearly, Theorem 4.6 and Corollary 4.5 imply Theorem 1.1. However, we will also have occasion to apply Theorem 4.6 in cases that are not 3-large; see Section 6. 
Let $R$ be a finitely generated $\mathbb{N}$-graded algebra over $\mathbb{C}$ of dimension $d$ with $R_{0}=\mathbb{C}$. Assume that $R$ is Gorenstein and normal. Let $K_{R}$ denote the canonical module of $R$ so that $K_{R}$ is graded isomorphic to $R$ with a degree shift; see Section 2.3. Let $\sigma$ be a homogeneous module generator of $K_{R}$, and let $X:=\operatorname{Spec} R$.

Let $I$ be the ideal of functions on $X \times X$ of the form $\sum_{i} f_{i}(x) \otimes h_{i}(y)$ such that $\sum_{i} f_{i}(x) h_{i}(x)=0$, and let $\Omega(X)=I / I^{2}$. Then $I$ is a homogeneous ideal, and the mapping $f \mapsto d f \in \Omega(X)$ defined by $f \mapsto f \otimes 1-1 \otimes f+I^{2}$ is degree-preserving. Note that $\Omega(X)$ is a graded $R$ module by multiplication on the first factor. Then the map $\left(h_{1}, \ldots, h_{d}\right) \mapsto d h_{1} \wedge \cdots \wedge d h_{d}$ is degree-preserving and maps $d$-tuples of homogeneous functions to homogeneous elements of $\wedge^{d}(\Omega(X))$. Then as $\sigma$ is a homogenous generator of $K_{R}$, it is homogeneous when considered as a section of $\wedge^{d}(\Omega(X))$. On the smooth locus $X_{\mathrm{sm}}$ of $X$, we can identify sections of $K_{R}$ with sections of $\wedge^{d}(\Omega(X))$. Since $X$ is normal, $\mathbb{C}\left[X_{\mathrm{sm}}\right]=\mathbb{C}[X]$. See [Har77, Section II.8] or CLS11, Section 8.0].

Let $f_{1}, \ldots, f_{d}$ be a homogeneous regular sequence for $R$ and let $d_{i}:=\operatorname{deg} f_{i}$. On $X_{\mathrm{sm}}$, we have $d f_{1} \wedge \cdots \wedge d f_{d}=\lambda \sigma$ where $\lambda$ is a regular function on $X_{\mathrm{sm}}$. Since $X$ is normal, $\lambda$ extends to a regular function on $X$, and as $\sigma$ and the $f_{i}$ are homogeneous, $\lambda$ is as well. Let $m:=\operatorname{deg} \lambda$. Note that if we calculate $\lambda$ in $K_{R}$, whose grading may be different than that on $\wedge^{d} \Omega(X)$, the ratio $\lambda$ remains the same.

Let $S=\mathbb{C}\left[f_{1}, \ldots, f_{d}\right]$. Since $R$ is Gorenstein, $R=S \otimes H$ where $H$ is a finite dimensional complex vector space whose Hilbert series has the form $1+\cdots+t^{r}$. As a graded module, $K_{R}=f_{1} \cdots f_{d} S \otimes H^{*}$ where $H^{*}$ has Hilbert series $1+\cdots+t^{-r}$. We demonstrate this with the following.

Lemma 4.7. The image of $d f_{1} \wedge \cdots \wedge d f_{d}$ in $K_{R}$ is $f_{1} \cdots f_{d} \otimes 1$ and hence has degree $\sum d_{i}$.

Proof. By the definition of $K_{R}$ we have that $K_{R}^{*}$ is calculated as the $n$th cohomology of the complex

$$
\begin{aligned}
0 \rightarrow R \rightarrow \oplus_{i} R\left[f_{i}^{-1}\right] \rightarrow \ldots & \rightarrow R\left[f_{2}^{-1}, \ldots, f_{d}^{-1}\right] \oplus \cdots \oplus R\left[f_{1}^{-1}, \ldots, f_{d-1}^{-1}\right] \\
& \rightarrow R\left[f_{1}^{-1}, \ldots, f_{d}^{-1}\right] \rightarrow 0,
\end{aligned}
$$

where all the mappings are inclusions up to a sign; see GW78, Section 2.1] and Hun07, Section 2.1]. Because $R=S \otimes H$, we may use the corresponding complex for $S$ and tensor each term with $H$. Hence, it is enough to consider the case where $R$ is replaced with $S$.

First consider the case of a polynomial ring in one variable $x$ of degree $e$. In this case, we have the complex

$$
0 \rightarrow \mathbb{C}[x] \rightarrow \mathbb{C}\left[x, x^{-1}\right] \rightarrow 0
$$

with cokernel $x^{-1} \mathbb{C}\left[x^{-1}\right]$. Taking the dual, we see that $K_{\mathbb{C}[x]} \simeq x \mathbb{C}[x]$ with the canonical grading. The mapping of $\Omega(\operatorname{Spec} \mathbb{C}[x])$ to $K_{\mathbb{C}[x]}$ sends the generator $d x$ of $\Omega(\operatorname{Spec} \mathbb{C}[x])$ to the generator $x$ of $K_{\mathbb{C}[x]}$ and hence preserves degrees. By an induction argument, it follows that in our case, $K_{R} \simeq f_{1} \cdots f_{d} \cdot S \otimes H^{*}$ with its canonical grading, and the image of $d f_{1} \wedge \cdots \wedge d f_{d}$ is $f_{1} \cdots f_{d} \otimes 1$.

Corollary 4.8. We have $r=m$ so that the isomorphism between sections of $K_{R}$ and $\wedge^{d} \Omega(X)$ is degree preserving. In particular, $\sigma$ has degree $\sum d_{i}-m$.

Proof. The degree of $\sigma$ in $\wedge^{d} \Omega(X)$ is $\sum d_{i}-m$ and its degree in $K_{R}$ is $\sum d_{i}-r$. Hence $r=m$. 
We are now ready to prove the main result of this section.

Proof of Theorem 4.6. Let $(V, G)$ be 1-large and assume that the shell $N$ is normal and that $\mathbb{C}\left[V \oplus V^{*}\right]^{G} /(\mu)^{G}$ is Gorenstein. Let $n=\operatorname{dim}_{\mathbb{C}} V$ and $g=\operatorname{dim}_{\mathbb{C}} G$. Let vol be the volume element on $V \oplus V^{*}$ so that, up to sign, vol $=d x_{1} \wedge \cdots \wedge d x_{n} \wedge$ $d y_{1} \wedge \cdots \wedge d y_{n}$ has degree $2 n$ and is $G$-invariant. Choosing a basis $A_{1}, \ldots, A_{g}$ for $\mathfrak{g}$, we let $\mu_{1}, \ldots, \mu_{g}$ denote the corresponding entries of the moment map with respect to the dual basis for $\mathfrak{g}^{*}$.

Note that as $(V, G)$ is 0-modular, the shell $N$ is a complete intersection by Sch95, Proposition 9.4]. In particular, the algebra of regular functions on the shell $\mathbb{C}[N]=\mathbb{C}\left[V \oplus V^{*}\right] /(\mu)$ is given by the quotient of the polynomial algebra $\mathbb{C}\left[V \oplus V^{*}\right]$ in $2 n$ variables by the complete intersection $(\mu)$ generated by $g$ quadratics, and hence has Hilbert series

$$
\operatorname{Hilb}_{\mathbb{C}[N]}(t)=\frac{\left(1-t^{2}\right)^{g}}{(1-t)^{2 n}}
$$

so that by Theorem 2.15, the $a$-invariant of $\mathbb{C}[N]$ is $2 g-2 n$. Hence, the generator $\sigma_{N}$ of the canonical module of $\mathbb{C}[N]$ has degree $2 n-2 g$. To describe $\sigma_{N}$ explicitly, if $f_{1}, \ldots, f_{2 n-g} \in \mathbb{C}\left[V \oplus V^{*}\right]$ are homogeneous, representing homogeneous elements of $\mathbb{C}[N]$, define

$$
\alpha_{N}\left(f_{1}, \ldots, f_{2 n-g}\right)=d f_{1} \wedge \cdots \wedge d f_{2 n-g} \wedge d \mu_{1} \wedge \cdots \wedge d \mu_{g} / \text { vol, }
$$

restricted to $N$. Note that $\alpha_{N}$ depends only on the elements of $\mathbb{C}[N]$ represented by the $f_{i}$. Because the $d \mu_{i}$ have degree 2 , the value of $\alpha_{N}\left(f_{1}, \ldots, f_{2 n-g}\right)$ is homogeneous of degree $\sum_{i} \operatorname{deg} f_{i}-(2 n-2 g)$. Then the form $\sigma_{N}$ is defined by

$$
\left.\left(d f_{1} \wedge \cdots \wedge d f_{2 n-g}\right)\right|_{N} / \sigma_{N}=\alpha_{N}\left(f_{1}, \ldots, f_{2 n-g}\right) .
$$

Note that $h \in G$ acts on $\sigma_{N}$ by the determinant of $h$ acting on $\mathfrak{g}^{*}$. Moreover, by Proposition $3.2(i v), \sigma_{N}$ does not vanish on the smooth locus of $N$.

Define

$$
\sigma_{N / / G}=\iota_{A_{1}} \ldots \iota_{A_{g}} \sigma_{N}
$$

where $\iota_{A_{i}}$ denotes contraction and the basis elements $A_{i}$ for $\mathfrak{g}$ are identified with the corresponding vector fields on $N$. Then $\sigma_{N / / G}$ is $G$-invariant and gives rise to an element of $\wedge^{2 n-2 g} \Omega\left((N / / G)_{\mathrm{sm}}\right)$. Because $(N / / G)_{\mathrm{pr}}=(N / / G)_{\mathrm{sm}}$, we have by Proposition $3.2(i v)$ and $(v)$ that the image under the orbit map $\pi: N \rightarrow N / / G$ of the smooth locus $N_{\text {sm }}$ contains $(N / / G)_{\text {sm }}$. Recalling that $N$ and $N / / G$ are normal, as $\sigma_{N}$ does not vanish on $N_{\mathrm{sm}}$, it follows that $\sigma_{N / / G}$ is a generator of $\wedge^{2 n-2 g} \Omega\left(N / / G_{\mathrm{sm}}\right)$, and hence of the canonical module of $\mathbb{C}[N]^{G}$. Because the vector fields $A_{i}$ have degree zero, the degree of $\sigma_{N / / G}$ is $2 n-2 g$. Noting that $\operatorname{dim}_{\mathbb{C}} N / / G=2 n-2 g$ completes the proof.

\section{The CASE OF $G^{0}$ A TORUS}

In this section, we prove Theorem 1.3. Because the shell $N$ depends only on $G^{0}$, we first consider the case that $G=G^{0}$ is a torus. Assume that $G=\left(\mathbb{C}^{\times}\right)^{\ell}$ for some positive integer $\ell$. Without loss of generality, assume that $V^{G}=\{0\}$ for simplicity, and by replacing $G$ with its image in $\operatorname{GL}(V)$, we assume that $(V, G)$ is faithful.

Choosing a basis for $V$ with respect to which the action of $G$ is diagonal, we may identify $V$ with $\mathbb{C}^{n}$ and describe the action of $G$ with a weight matrix $A=\left(a_{i j}\right) \in$ 
$\mathbb{Z}^{\ell \times n}$. Specifically, in coordinates $\left(z_{1}, \ldots, z_{n}\right)$ with respect to our basis, the action of $G$ is given by

$$
\left(t_{1}, \ldots, t_{\ell}\right) \cdot\left(z_{1}, \ldots, z_{n}\right):=\left(z_{1} \prod_{i=1}^{\ell} t_{i}^{a_{i 1}}, \ldots, z_{n} \prod_{i=1}^{\ell} t_{i}^{a_{i n}}\right) .
$$

for $\left(t_{1}, \ldots, t_{\ell}\right) \in G=\left(\mathbb{C}^{\times}\right)^{\ell}$. Choosing the dual basis for $V^{*}$ and using coordinates $\left(z_{1}, \ldots, z_{n}, w_{1}, \ldots, w_{n}\right)$ with respect to the concatenated basis for $V \oplus V^{*}$, the weight matrix for the action of $G$ on $V \oplus V^{*}$ is $(A \mid-A)$. Using the symplectic form $\omega=\sqrt{-1} / 2 \sum_{j=1}^{n} d z_{j} \wedge d \bar{z}_{j}$ on $V$, and identifying $\mathfrak{k}^{*}$ with $\mathbb{R}^{\ell}$ via the coordinates for $\left(\mathbb{C}^{\times}\right)^{\ell}$ used above, the real moment map $J: V \rightarrow \mathfrak{k}^{*}$ is given by the component functions

$$
J_{i}(\boldsymbol{z}, \overline{\boldsymbol{z}})=\sum_{j=1}^{n} a_{i j} z_{j} \overline{z_{j}}, \quad i=1, \ldots, \ell .
$$

It follows that the complexified moment map $\mu: V \oplus V^{*} \rightarrow \mathfrak{g}^{*}$ is given by the component functions

$$
\mu_{i}(\boldsymbol{z}, \boldsymbol{w})=\sum_{j=1}^{n} a_{i j} z_{j} w_{j}, \quad i=1, \ldots, \ell .
$$

See [FHS13, HIP09, HS15] for more details.

Using Weh92, Lemma 2], we may reduce to the case that $(V, G)$ is stable; see also [FHS13, Section 3] and [HS14, Lemma 3]. Specifically, we have the following.

Lemma 5.1. Let $G=\left(\mathbb{C}^{\times}\right)^{\ell}$ and let $V$ be a faithful $G$-module with $V^{G}=\{0\}$. Then there is a submodule $V^{\prime}$ of $V$ such that $\left(V^{\prime}, G^{\prime}\right)$ is stable and faithful, where $G^{\prime}$ is the quotient of $G$ by the subgroup that acts trivially on $V^{\prime}$. Moreover, the real shell and the real and complex symplectic quotients corresponding to $(V, G)$ and $\left(V^{\prime}, G^{\prime}\right)$ coincide.

Proof. If $(V, G)$ is stable, then there is nothing to prove, so assume not. Then $V / / G$ has complex dimension strictly less than $n-\ell$. By the Kempf-Ness homeomorphism Sch89, Corollary 4.7], the real symplectic quotient $M_{0}=M / K$ has real dimension less than $2 n-2 \ell$ from which it follows that the real shell $M$ has real dimension less than $2 n-\ell$. Considering the moment map in Equation (5.1), it follows that there are coordinates $z_{i}$ that vanish on $M$; again by the Kempf-Ness homeomorphism, these coordinates do not appear in any invariant polynomial. By Weh92, Lemma 2], restricting to the subspace of $V^{\prime}$ of $V$ on which all such coordinates vanish yields a stable torus-module $\left(V^{\prime}, G^{\prime}\right)$ such that $V / / G=V^{\prime} / / G^{\prime}$. Moreover, as the real shell $M$ is contained in $V^{\prime}$, restricting to $\left(V^{\prime}, G^{\prime}\right)$ does not change the real symplectic quotient $M_{0}$. As the complex symplectic quotient is defined in Definition 2.5 to be the complexification of the real symplectic quotient, it also does not change the complex symplectic quotient.

Note that Lemma 5.1 can be understood as an application of the Luna-Richardson Theorem, LR79, Corollary 4.4]. Specifically, if $H$ is the principal isotropy group of $V$, then inclusion yields an isomorphism $\mathbb{C}\left[V^{H}\right]^{N_{G}(H) / H}=\mathbb{C}\left[V^{H}\right]^{G / H} \simeq \mathbb{C}[V]^{G}$. Because $H$ acts with no closed orbits on a $G$-complement to $V^{H}$, the invariants only involve coordinates of $V^{H}$. By construction, $\left(V^{H}, G / H\right)$ has FPIG and hence is stable, and the real shell $M$ is contained in $V^{H}$. 
Reducing to the case of $(V, G)$ stable and faithful, we can now demonstrate the following. See also Bul18, Theorem 2.2], where it was demonstrated independently and simultaneously without the hypothesis of stable that $N$ is normal if and only if it is irreducible.

Lemma 5.2. Suppose that $G=\left(\mathbb{C}^{\times}\right)^{\ell}$ is a torus and that $(V, G)$ is stable and faithful. Then both the shell $N$ and the complex symplectic quotient $N / / G$ are normal varieties.

Proof. By [HS13, Theorem 3.2], Vin86, Proposition 1], $(V, G)$ is 1-large, and by Lemma 2.2 it follows that $\operatorname{dim}_{\mathbb{C}} V>\operatorname{dim}_{\mathbb{C}} G$. The shell $N$ is a complete intersection and Cohen-Macaulay by Proposition 3.2 $(i)$. By HS13, Corollary 4.3], $(\mu)$ is radical and hence the ideal of $N$. Because row-reducing the weight matrix $A$ over $\mathbb{Z}$ corresponds to choosing a different basis for $\mathfrak{g}^{*}$ and corresponding coordinates for $G$, we may assume that the weight matrix is in the form $A=(D \mid C)$ where $D$ is diagonal and has strictly negative entries.

Using Equation (5.2), we express the Jacobian matrix of the moment map $\mu: V \oplus$ $V^{*} \rightarrow \mathfrak{g}^{*}$ as

$$
d \mu=\left(D_{1}|\cdots| D_{2} \mid \cdots\right)
$$

where $D_{1}$ is the $\ell \times \ell$ diagonal matrix with $(i, i)$-entry $a_{i i} w_{i}, D_{2}$ is the $\ell \times \ell$ diagonal matrix with $(i, i)$-entry $a_{i i} z_{i}$, and the $\cdots$ indicate $\ell \times(n-\ell)$ blocks. As $a_{i i} \neq 0$ for $i \leq \ell$, it follows from Proposition $3.2(i v)$ that $(\boldsymbol{z}, \boldsymbol{w}) \in V \oplus V^{*}$ is a smooth point of $N$ unless $z_{j}=w_{j}=0$ for some $j$. Therefore, $N$ is smooth in codimension 1 . Then by Serre's Criterion [Mat89, Theorem 23.8], $N$ is Cohen-Macaulay and regular in codimension 1 , hence normal.

Remark 5.3. Note that there is a mistake in the proof of [HS15, Theorem 3], where it is incorrectly assumed that the torus representation is 2-large. Lemma 5.2 offers an alternate proof that corrects this mistake.

We now have the following.

Proposition 5.4. Suppose that $G=\left(\mathbb{C}^{\times}\right)^{\ell}$ is a torus and that $(V, G)$ is stable and faithful. Then both the shell $N$ and the complex symplectic quotient $N / / G$ have rational singularities.

Proof. If the symplectic quotient is a point, then the result is trivial, so assume not. As above, we assume without loss of generality that $V^{G}=\{0\}$ and choose coordinates with respect to which $A=(D \mid C) \in \mathbb{Z}^{\ell \times n}, \ell<n$, with $D$ diagonal having strictly negative entries on the diagonal. Recalling that $N$ is a complete intersection with $(\mu)$ generated by $\ell$ quadratics, the Hilbert series of $\mathbb{C}[N]=\mathbb{C}[V \oplus$ $\left.V^{*}\right] /(\mu)$ is given by

$$
\operatorname{Hilb}_{\mathbb{C}[N]}(t)=\frac{\left(1-t^{2}\right)^{\ell}}{(1-t)^{2 n}}
$$

so that the $a$-invariant of $N$ is $2(\ell-n)$. In particular, $\ell<n$ implies that the $a$-invariant is negative. With this, the proof is by induction on $\ell$.

If $\ell=1$, then the moment map $\mu: V \oplus V^{*} \rightarrow \mathfrak{g}^{*}$ is given by the single function

$$
\mu(\boldsymbol{z}, \boldsymbol{w})=\sum_{j=1}^{n} a_{j} z_{j} w_{j}
$$


with Jacobian

$$
d \mu(\boldsymbol{z}, \boldsymbol{w})=\left(a_{1} w_{1}, \ldots, a_{n} w_{n}, a_{1} z_{1}, \ldots, a_{n} w_{n}\right) .
$$

As $V^{G}=\{0\}$, each $a_{j} \neq 0$ so that $d \mu$ has rank 1 away from the origin. It follows that $N$ is Cohen-Macaulay, normal, has negative $a$-invariant, and is regular away from the origin so that, by a theorem of Flenner and Watanabe [Fle81, Satz 3.1], Wat83, Theorem 2.2], Hun98, Theorem 9.2], $N$ has rational singularities.

Now assume that $G$ has complex dimension $\ell$ and that the result holds for representations of tori of dimension less than $\ell$. We have established that $\mathbb{C}[N]$ is normal, Cohen-Macaulay, and has negative $a$-invariant, so again by the theorem of Flenner and Watanabe, it suffices to show that $N$ has rational singularities away from the origin. Let $p \in N$ be a nonzero point, and then $p$ has a nonzero coordinate. By switching $V$ and $V^{*}$ and permuting the bases for $V$ and $V^{*}$, we may assume that $p$ has nonzero $z_{1}$-coordinate. Recalling that $V^{G}=\{0\}$ and row-reducing $A$ (i.e., changing bases for $\mathfrak{g}^{*}$ ) results in a weight matrix $A=(D \mid C)$ such that $a_{11} \neq 0$ and $a_{1 i}=0$ for $i=2, \ldots, \ell$. Then

$$
\mu_{1}(\boldsymbol{z}, \boldsymbol{w})=a_{11} z_{1} w_{1}+\sum_{j=\ell+1}^{n} a_{1 j} z_{j} w_{j},
$$

and each $\mu_{i}$ for $i>1$ involves neither $z_{1}$ nor $w_{1}$. Localizing to the set $z_{1} \neq 0$, we adjoin an inverse to $z_{1}$ and express the condition $\mu_{1}=0$ as

$$
w_{1}=-\sum_{j=\ell+1}^{n} \frac{a_{1 j} z_{j} w_{j}}{a_{11} z_{1}}
$$

eliminating $w_{1}$. Then on the set $z_{1} \neq 0, N$ is isomorphic to the product of $\mathbb{C}$ with the shell associated to the $(\ell-1)$-dimensional torus action with weight matrix formed by removing the first row and column from $A$. This has rational singularities by the inductive hypothesis, completing the proof that $N$ has rational singularities. Then $N / / G$ has rational singularities by a theorem of Boutot Bou87.

As described above, if $(V, G)$ is not stable, then the corresponding complex symplectic quotient coincides with that of a stable torus representation so that the symplectic quotient still has rational singularities. However, the shell $N$ may fail to have rational singularities.

Example 5.5. Let $G=\left(\mathbb{C}^{\times}\right)^{n}$ act on $V=\mathbb{C}^{n}$ with weight matrix given by the identity matrix. Then the only closed orbit is the origin so that $(V, G)$ is not stable. Noting that $\ell=n$ in Equation (5.3), one observes that the $a$-invariant of the shell $N$ is zero so that, again by the theorem of Flenner and Watanabe Fle81, Satz 3.1], Wat83, Theorem 2.2], Hun98, Theorem 9.2], $N$ does not have rational singularities.

In order to apply Theorem 4.6 to this case, we will need the following.

Lemma 5.6. Let $G=\left(\mathbb{C}^{\times}\right)^{\ell}$ be a torus and let $(V, G)$ be 1-modular. Then $N / / G$ is not smooth.

Proof. As above, assume without loss of generality that $V^{G}=\{0\}$ and $V$ is faithful, choose coordinates $z_{1}, \ldots, z_{n}$ on $V \simeq \mathbb{C}^{n}$ with respect to which the action of $G$ is diagonal, and let $w_{1}, \ldots, w_{n}$ be the dual coordinates. Because $V$ is 1-modular, $\ell \leq n-1$ by Lemma 2.2 . 
Assume for simplicity that the characters corresponding to $z_{1}, \ldots, z_{\ell}$ are linearly independent in the character group $X(G)$ of $G$. Then there are $n-\ell$ indices $i$ such that $z_{i} w_{i}$ does not vanish on $N$. For each index $k>\ell$, there is a minimal invariant of the form $z_{k}^{a_{k}} w_{k}^{b_{k}} \prod_{i=1}^{\ell} z_{i}^{a_{i}} \prod_{i=1}^{\ell} w_{j}^{b_{j}}$ where the $a_{i}$ and $b_{j}$ are nonnegative and $a_{i} b_{i}=0$ for all $i$. Dually, there is a minimal invariant $w_{k}^{a_{k}} z_{k}^{b_{k}} \prod_{i=1}^{\ell} w_{i}^{a_{i}} \prod_{i=1}^{\ell} z_{j}^{b_{j}}$. Hence, there are at least $3(n-\ell)$ minimal generators, while $\operatorname{dim}_{\mathbb{C}} N / / G \leq 2(n-\ell)$, so that $N / / G$ is not smooth.

If $(V, G)$ is 1-large and $G^{0}$ is a torus, then for any symplectic slice representation $\left(W \oplus W^{*}, L\right),\left(W, L^{0}\right)$ is 1-modular by Corollary [3.16] Hence, applying Lemma 5.6 to the local model $N_{S} / / L$ of $N / / G$ corresponding to each $\left(W, L^{0}\right)$ as well as Proposition 3.19, we have that $(N / / G)_{\mathrm{pr}}=(N / / G)_{\mathrm{sm}}$ in this case. Note that if $(V, G)$ is not 1-large, then $N / / G$ may not be the complex symplectic quotient of Definition 2.5.

Proof of Theorem 1.3. Assume that $G$ is a reductive group such that $G^{0}$ is a torus. Let $V$ be a $G$-module, and let $V=V_{1} \oplus V_{2}$ where $V_{2}=V^{G^{0}}$. Then letting $M$ denote the real shell associated to $\left(V_{1}, G^{0}\right)$ the real symplectic quotient associated to $(V, G)$ is given by $\left(M \times V_{2}\right) / K=\left(\left(M / K^{0}\right) \times V_{2}\right) /\left(K / K^{0}\right)$. By Lemma 5.1, we may replace $\left(V_{1}, G^{0}\right)$ with a faithful stable torus module $\left(V_{1}^{\prime},\left(G^{0}\right)^{\prime}\right)$ such that the real shell $M$ and the real and complex symplectic quotients of $\left(V_{1}, G^{0}\right)$ and $\left(V_{1}^{\prime},\left(G^{0}\right)^{\prime}\right)$ coincide. Let $N$ denote the complex shell associated to $\left(V_{1}^{\prime},\left(G^{0}\right)^{\prime}\right)$ and then the corresponding complex symplectic quotient is given by $N / /\left(G^{0}\right)^{\prime}$. By Proposition 5.4 both $N$ and $N / /\left(G^{0}\right)^{\prime}$ have rational singularities. Similarly, Boutot's theorem Bou87 implies that $N / / G$ has rational singularities.

Recall that the Hilbert series of regular functions on the real and complex symplectic quotients coincide. The complex symplectic quotient $N / /\left(G^{0}\right)^{\prime}$ is then graded Gorenstein by [HHS15, Theorem 1.3 and Corollary 1.8]. Then by Theorem 2.14. $N / /\left(G^{0}\right)^{\prime}$ has symplectic singularities. In the case $G=G^{0}$, it follows that the complex symplectic quotient $N / /\left(G^{0}\right)^{\prime}$ associated to $(V, G)$ is graded Gorenstein with symplectic singularities, completing the proof in this case. Otherwise, $\left(N / /\left(G^{0}\right)^{\prime}\right) \times\left(V_{2} \oplus V_{2}^{*}\right)$ has symplectic singularities so that by Bea00, Proposition $2.4]$, the symplectic quotient $\left(\left(N / /\left(G^{0}\right)^{\prime}\right) \times\left(V_{2} \oplus V_{2}^{*}\right)\right) /\left(G / G^{0}\right)$ associated to $(V, G)$ has symplectic singularities. Finally, let $\sigma_{\left(N \times V_{2} \oplus V_{2}^{*}\right) / /\left(G^{0}\right)^{\prime}}$ denote the generator of the canonical module of $\mathbb{C}\left[N \times V_{2} \oplus V_{2}^{*}\right]^{\left(G^{0}\right)^{\prime}}$ constructed in the proof of Theorem 4.6. An element $g \in G$ acts on $\sigma_{N \times V_{2} \oplus V_{2}^{*}}$ by the determinant of the action on $\mathfrak{g}^{*}$ and acts on $A_{1} \wedge \cdots \wedge A_{\ell}$ by the determinant of the action on $\mathfrak{g}$; as these determinants are inverses, $\sigma_{\left(N \times V_{2} \oplus V_{2}^{*}\right) / /\left(G^{0}\right)^{\prime}}$ is in fact $G$-invariant. Hence $\mathbb{C}\left[N \times V_{2} \oplus V_{2}^{*}\right]^{\left(G^{0}\right)^{\prime}}$ and $\mathbb{C}\left[N \times V_{2} \oplus V_{2}^{*}\right]^{G}$ have the same $a$-invariant and dimension, implying that $\mathbb{C}\left[N \times V_{2} \oplus V_{2}^{*}\right]^{G}$ is graded Gorenstein and completing the proof.

\section{The CASE OF $K=\mathrm{SU}_{2}$}

In this section, we prove Theorem 1.4 Throughout this section, we let $K=\mathrm{SU}_{2}$ so that $G=\mathrm{SL}_{2}(\mathbb{C})$. In this case, every irreducible unitary $K$-module is isomorphic to $\left(R_{d}, \mathrm{SU}_{2}\right)$ for some $d \geq 1$ where $R_{d}$ denotes the set of binary forms of degree $d$; similarly, every irreducible $\mathrm{SL}_{2}(\mathbb{C})$-module is isomorphic to some $\left(R_{d}, \mathrm{SL}_{2}(\mathbb{C})\right.$ ), see [Wig59, Section 15.6] or [Ber07, Section 4]. Note that $R_{d} \simeq S^{d} \mathbb{C}^{2}$ with the canonical $\mathrm{SL}_{2}(\mathbb{C})$-action. 
The proper reductive subgroups of $\mathrm{SL}_{2}(\mathbb{C})$ are finite, $\mathbb{C}^{\times}$, and the normalizer of $\mathbb{C}^{\times}$, with the connected component of the identity in the latter equal to $\mathbb{C}^{\times}$. Hence, if $\left(V, \mathrm{SL}_{2}(\mathbb{C})\right)$ is 2-large, then for each symplectic slice representation $\left(W \oplus W^{*}, L\right)$ such that $L$ is not principal, we have that $(W, L)$ is 1-modular by Corollary 3.16 If $L=\mathrm{SL}_{2}(\mathbb{C})$, then $\left(W, \mathrm{SL}_{2}(\mathbb{C})\right)=\left(V, \mathrm{SL}_{2}(\mathbb{C})\right)$ and the corresponding $N_{S} / / L$ is singular by [HSS15, Lemma 2.3]. Otherwise, $N_{S} / / L$ is singular by Lemma 5.6 and Proposition 3.19 so that $(N / / G)_{\mathrm{pr}}=(N / / G)_{\mathrm{sm}}$.

By Sch95, Theorem 11.9], we have that the nontrivial finite-dimensional complex representations $\bigoplus_{d \geq 1}\left(R_{d}\right)^{\oplus m_{d}}$ of $\mathrm{SL}_{2}(\mathbb{C})$ that are not 2-large are the following:

$$
R_{1}^{\oplus k} \text { for } 1 \leq k \leq 3, \quad R_{2}, \quad R_{2}^{\oplus 2}, \quad R_{2} \oplus R_{1}, \quad R_{3}, \quad R_{4} .
$$

Hence, all other nontrivial $\mathrm{SL}_{2}(\mathbb{C})$-modules satisfy the hypotheses of Theorem 1.1 The cases listed above are exactly those treated in [Bec10, Theorem 1.2] using a different approach; we will apply Becker's results in some cases but also indicate alternative methods to treat these cases.

By [HSS15, Theorem 1.6], the real symplectic quotients corresponding to the following representations are each graded regularly symplectomorphic to a linear symplectic orbifold:

$$
R_{1}^{\oplus k} \text { for } k=1,2, \quad R_{2}, \quad R_{3}, \quad R_{4},
$$

That is, for each $\mathrm{SL}_{2}(\mathbb{C})$-module $V$ listed above, the real regular functions $\mathbb{R}\left[M_{0}\right]$ on the real symplectic quotient $M_{0}$ are graded isomorphic to $\mathbb{R}\left[W \oplus W^{*}\right]^{H}$ where $H$ is a finite group and $W$ is a unitary $H$-module (considered as its underlying real vector space). It is an immediate consequence that the corresponding complex symplectic quotients are graded isomorphic to $\left(W_{\mathbb{C}} \oplus W_{\mathbb{C}}^{*}\right) / H$ where $W_{\mathbb{C}}=W \otimes_{\mathbb{R}} \mathbb{C}$. Such a quotient has symplectic singularities and is graded Gorenstein by Theorem 2.16. Hence, Theorem 1.4 holds for all nontrivial $\mathrm{SL}_{2}(\mathbb{C})$-modules $V$ such that $V^{\mathrm{SL}_{2}(\mathbb{C})}=\{0\}$ except for $R_{1}^{\oplus 3}, R_{2}^{\oplus 2}$, and $R_{2} \oplus R_{1}$. We will consider these three representations individually below, demonstrating Theorem 1.4 in each case as well as the stronger fact that the shell $N$ has rational singularities.

Each of the orbifold cases listed above is 1-large except for $R_{1}, R_{1}^{\oplus 2}$, and $R_{2}$. In the case of $R_{2},(J)$ is real by AGJ90, Example 7.14] so that Lemma 2.8 applies. That is, our definition of the complex symplectic quotient coincides with those of Equations (2.4) and (2.5). This, however, is not the case for $R_{1}$ and $R_{1}^{\oplus 2}$; see Examples 2.6 and 2.7 above.

6.1. $R_{2}^{\oplus 2}$. Let $V=R_{2}^{\oplus 2}$ and recall that $\left(R_{2}, \mathrm{SL}_{2}(\mathbb{C})\right)$ is isomorphic to the adjoint representation of $\mathrm{SL}_{2}(\mathbb{C})$. Then the action of $\mathrm{SL}_{2}(\mathbb{C})$ on $V$ is not effective, as the negative identity acts trivially. We have $\mathrm{SL}_{2}(\mathbb{C}) /\{ \pm \mathrm{id}\} \simeq \mathrm{SO}_{3}(\mathbb{C})$, and $V$ is isomorphic to $W^{\oplus 2}$ where $W$ is the standard representation of $\mathrm{SO}_{3}(\mathbb{C})$ on $\mathbb{C}^{3}$, i.e. $V \oplus V^{*}$ is isomorphic to $W^{\oplus 4}$.

Recall [HS13, Theorem 3.4] that $V$ is 1-large so that the ideal $(J)$ generated by the moment map in $\mathbb{R}\left[W^{\oplus 2}\right]$ is real, hence the ideal $(\mu)$ in $\mathbb{C}\left[W^{\oplus 4}\right]$ is radical. In CHS16, Theorem 5.1], it is demonstrated that the variety of the moment map associated to $V=W^{\oplus 2}$ has rational singularities. Note that this theorem considers the corresponding real variety, but the proof implicitly appeals to the complexification and hence is identical for the complex variety. Hence, by the theorem of Boutot Bou87, the complex symplectic quotient $N / / \mathrm{SO}_{3}(\mathbb{C})=N / / \mathrm{SL}_{2}(\mathbb{C})$ has rational singularities. 
Similarly, in CHS16, Section 6.2], the Hilbert series of the ring $\mathbb{R}\left[M_{0}\right]$ of regular functions on the real symplectic quotient is computed to be

$$
\operatorname{Hilb}_{\mathbb{R}\left[M_{0}\right]}(t)=\frac{1+4 t^{2}+4 t^{4}+t^{6}}{\left(1-t^{2}\right)^{6}} .
$$

This of course coincides with the Hilbert series of the regular functions $\mathbb{R}\left[M_{0}\right] \otimes_{\mathbb{R}} \mathbb{C}$ on the complex symplectic quotient $N / / \mathrm{SL}_{2}(\mathbb{C})$. As

$$
\operatorname{Hilb}_{\mathbb{R}\left[M_{0}\right]}(1 / t)=\frac{t^{6}\left(1+4 t^{2}+4 t^{4}+t^{6}\right)}{\left(1-t^{2}\right)^{6}}=(-1)^{6} t^{6} \operatorname{Hilb}_{\mathbb{R}\left[M_{0}\right]}(t),
$$

Equation (2.6) is satisfied with negative $a$-invariant equal to 6, the Krull dimension, so that the complex symplectic quotient is graded Gorenstein.

We have that $N / / \mathrm{SL}_{2}(\mathbb{C})$ is Gorenstein with rational singularities, and by Corollary 3.18 , the smooth locus $\left(N / / \mathrm{SL}_{2}(\mathbb{C})\right)_{\text {sm }}$ admits a regular symplectic form. By Theorem 2.14, $N / / \mathrm{SL}_{2}(\mathbb{C})$ has symplectic singularities. It follows that Theorem 1.4 holds in this case.

6.2. $R_{1}^{\oplus 3}$. Suppose that $V=R_{1}^{\oplus 3}$ and note that $\left(V, \mathrm{SL}_{2}(\mathbb{C})\right)$ is 1-large. Then $V \oplus V^{*}$ has complex dimension 12 so that as the shell $N$ is a complete intersection by [Sch95, Proposition 9.4], $N$ has complex dimension 9. In addition, $V \oplus V^{*}$ is isomorphic to $R_{1}^{\oplus 6}$ and has a linear action of $\mathrm{GL}_{6}(\mathbb{C})$ commuting with that of $\mathrm{SL}_{2}(\mathbb{C})$. The quotient $\left(R_{1}^{\oplus 6}\right) / / \mathrm{SL}_{2}(\mathbb{C})$ is isomorphic to the subspace of $\wedge^{2} \mathbb{C}^{6}$ of 2 -forms of rank at most two. There are two $\mathrm{GL}_{6}(\mathbb{C})$-orbits in the quotient, one of them being the origin. Now, a point $v \in R_{1}^{\oplus 6}$ is in the null cone $\mathcal{N}=\mathcal{N}\left(R_{1}^{\oplus 6}\right)$ if and only if the $\mathrm{SL}_{2}(\mathbb{C})$-isotropy group of the point is nontrivial, i.e., if and only if the six components of $v$ do not span $R_{1}$. It follows that $\mathcal{N}$ has complex dimension 7 and hence intersects $N$ in complex codimension at least 2. By Proposition 3.2 as $(N \backslash \mathcal{N}) \subset N_{\text {sm }}$, it follows that $N$ is normal. Similarly, $N / / \mathrm{SL}_{2}(\mathbb{C}) \subset \wedge^{2} \mathbb{C}^{6}$ is smooth except at the orbit of the origin. However, $\operatorname{dim}_{\mathbb{C}} N / / \mathrm{SL}_{2}(\mathbb{C})=6$ so that by Theorem 2.13 and Corollary 3.18, $N / / \mathrm{SL}_{2}(\mathbb{C})$ has symplectic singularities. In fact, the shell $N$ has rational singularities; this can be verified by a computation similar to, though simpler than, that given in Lemma 6.1 below.

Similarly, as $\left(V, \mathrm{SL}_{2}(\mathbb{C})\right)$ is 1-large and $N$ is normal, it follows from Theorem 4.6 that $N / / \mathrm{SL}_{2}(\mathbb{C})$ is graded Gorenstein. Alternatively, we have computed the Hilbert series of the regular functions on the (real or complex) symplectic quotient to be

$$
\operatorname{Hilb}_{\mathbb{C}\left[N / / \mathrm{SL}_{2}(\mathbb{C})\right]}(t)=\frac{1+9 t^{2}+9 t^{4}+t^{6}}{\left(1-t^{2}\right)^{6}} .
$$

The $a$-invariant is $-6=-\operatorname{dim}_{\mathbb{C}} \mathbb{C}\left[N / / \mathrm{SL}_{2}(\mathbb{C})\right]$ implying by Theorem 2.15 that $\mathbb{C}\left[N / / \mathrm{SL}_{2}(\mathbb{C})\right]$ is graded Gorenstein.

6.3. $R_{2} \oplus R_{1}$. Let $V=R_{2} \oplus R_{1}$, and then $\left(V, \mathrm{SL}_{2}(\mathbb{C})\right)$ is 1-large so that Lemma 2.8 applies. An explicit description of the generators and relations of the regular functions on the complex symplectic quotient $\mathbb{C}\left[V \oplus V^{*}\right]^{\mathrm{SL}_{2}(\mathbb{C})} /(\mu)^{\mathrm{SL}_{2}(\mathbb{C})}$ is computed in [Bec10, Proposition 5.3], which is used in [Bec10, Proposition 5.5] to demonstrate that $N / / \mathrm{SL}_{2}(\mathbb{C})$ is a symplectic variety. Moreover, one may use this description to compute the Hilbert series

$$
\operatorname{Hilb}_{\mathbb{C}\left[N / / \mathrm{SL}_{2}(\mathbb{C})\right]}(t)=\frac{1+2 t^{2}+3 t^{3}+2 t^{4}+2 t^{5}+3 t^{6}+2 t^{7}+t^{9}}{\left(1-t^{2}\right)^{2}\left(1-t^{3}\right)\left(1-t^{6}\right)} .
$$


Using this and Theorem 2.15, one computes that the $a$-invariant is -4 and the complex dimension is 4 so that $N / / \mathrm{SL}_{2}(\mathbb{C})$ is graded Gorenstein.

Let us indicate an alternative method of demonstrating that $N / / \mathrm{SL}_{2}(\mathbb{C})$ is a symplectic variety and computing the Hilbert series. First, we observe that in this case, a variation on the argument given in [CHS16, Theorem 5.1] can be used to prove that the shell $N$ itself has rational singularities.

Lemma 6.1. The shell $N$ associated to $\left(R_{2} \oplus R_{1}, \mathrm{SL}_{2}(\mathbb{C})\right)$ has rational singularities.

Proof. Let $V=R_{2} \oplus R_{1}$. We use coordinates for $V \oplus V^{*}$ transforming by weights of $\mathbb{C}^{\times} \leq \mathrm{SL}_{2}(\mathbb{C})$. For $R_{2}$ we have $z_{2}, z_{0}$ and $z_{-2}$ (of weights indicated by the subscripts), and for $R_{2}^{*}$ we similarly have $z_{2}^{\prime}, z_{0}^{\prime}$ and $z_{-2}^{\prime}$. For $R_{1}$ we have $x$ of weight 1 and $y$ of weight -1 , and similarly $x^{\prime}$ and $y^{\prime}$ for $R_{1}^{*}=R_{1}$. Then the coordinate functions of the moment map are given by

$$
\begin{aligned}
& \mu_{1}=x x^{\prime}+z_{0} z_{2}^{\prime}-z_{2} z_{0}^{\prime}, \\
& \mu_{2}=x y^{\prime}+y x^{\prime}+2\left(z_{-2} z_{2}^{\prime}-z_{2} z_{-2}^{\prime}\right), \\
& \mu_{3}=y y^{\prime}+z_{-2} z_{0}^{\prime}-z_{0} z_{-2}^{\prime} .
\end{aligned}
$$

As $\left(V, \mathrm{SL}_{2}(\mathbb{C})\right)$ is 1-large, $(\mu)$ is radical and $N$ is a complete intersection. In particular, $\mathbb{C}\left[V \oplus V^{*}\right] /(\mu)$ is Cohen-Macaulay with Hilbert series $\left(1-t^{2}\right)^{3} /(1-t)^{10}$, hence the $a$-invariant is -4 . A computation of $d \mu$ demonstrates that $N$ is smooth in codimension 1 and hence normal. With this, by the theorem of Flenner and Watanabe [Fle81, Satz 3.1], Wat83, Theorem 2.2], Hun98, Theorem 9.2], it is sufficient to show that $N$ has rational singularities away from the origin. Because $\mathrm{SL}_{2}(\mathbb{C})$ acts on $N$ as isomorphisms, it is sufficient to show that each orbit contains a point at which $N$ has rational singularities.

At a point where $x$ or $y$ does not vanish, we can by applying an element of $\mathrm{SL}_{2}(\mathbb{C})$ assume that both $x$ and $y$ do not vanish. Localizing at such points, we solve $\mu_{1}=0$ for $x^{\prime}$ and $\mu_{3}=0$ for $y^{\prime}$, and then the equation $\mu_{2}=0$ can be written

$$
-2 u v^{\prime}+2 v u^{\prime}=0
$$

where $u=z_{2} y / x-z_{0} / 2, v=z_{-2} x / y-z_{0} / 2, u^{\prime}=z_{2}^{\prime} y / x-z_{0}^{\prime} / 2$, and $v^{\prime}=z_{-2}^{\prime} x / y-$ $z_{0}^{\prime} / 2$. The corresponding hypersurface is singular only at the origin and hence has rational singularities by Flenner-Watanabe. The same argument applies near points where $x^{\prime}$ or $y^{\prime}$ does not vanish.

Near a nonzero point where $x, y, x^{\prime}$, and $y^{\prime}$ vanish, either some $z_{i}$ or some $z_{i}^{\prime}$ is nonzero. Assume some $z_{i}^{\prime} \neq 0$, and then by moving within an orbit, we may assume that $z_{0}^{\prime} \neq 0$. Localizing at such points, we solve $\mu_{1}=0$ for $z_{2}$ and $\mu_{3}=0$ for $z_{-2}$ and then express $\mu_{2}=0$ as

$$
x^{\prime} s+y^{\prime} t=0
$$

where $s=y-2 x z_{-2}^{\prime} / z_{0}^{\prime}$ and $t=x-2 y z_{2}^{\prime} / z_{0}^{\prime}$. We again have that this hypersurface has an isolated singularity at the origin and negative $a$-invariant and hence rational singularities by Flenner-Watanabe. The same argument again applies near points where some $z_{i}$ is nonzero, completing the proof.

Finally, we describe a direct computation of the Hilbert series of $N / / G$ given in Equation (6.1). Consider $\mathfrak{g}$ as the quadratic equations defining the shell $N$. We again have that the elements of $\mathfrak{g}$ are a regular sequence. Let $S$ denote $\mathbb{C}\left[V \oplus V^{*}\right]$, and then we have an exact sequence

$$
0 \rightarrow S \simeq S \otimes \wedge^{3} \mathfrak{g} \rightarrow S \otimes \wedge^{2} \mathfrak{g} \rightarrow S \otimes \mathfrak{g} \rightarrow S \rightarrow S / \mathfrak{g} \rightarrow 0 .
$$


Taking $G=\mathrm{SL}_{2}(\mathbb{C})$-invariants yields

$$
0 \rightarrow S^{G} \rightarrow\left(S \otimes \wedge^{2} \mathfrak{g}\right)^{G} \rightarrow(S \otimes \mathfrak{g})^{G} \rightarrow S^{G} \rightarrow(S / \mathfrak{g})^{G} \rightarrow 0 .
$$

Now, the elements of $\mathfrak{g}$ are in degree 2, the elements of $\wedge^{2} \mathfrak{g}$ are in degree 4 , and $\wedge^{3} \mathfrak{g}$ is in degree 6 . Let $a(t)$ denote the Hilbert series of $S^{G}$ and let $b(t)$ denote the Hilbert series for the occurrences of $\mathfrak{g}$ in $S$. Then the Hilbert series of $(S / \mathfrak{g})^{G}$ is

$$
\operatorname{Hilb}_{\mathbb{C}\left[N / / \mathrm{SL}_{2}(\mathbb{C})\right]}(t)=a(t)-t^{2} b(t)+t^{4} b(t)-t^{6} a(t) .
$$

Now one can use the program Lie vLCL92 to calculate $a(t)$ and $b(t)$ to any degree. Going up to degree 14, we found that $\operatorname{Hilb}_{\mathbb{C}\left[N / / \mathrm{SL}_{2}(\mathbb{C})\right]}(t)$ is the rational function given by Equation (6.1). Using classical invariant theory, one can compute that $\mathbb{C}\left[N / / \mathrm{SL}_{2}(\mathbb{C})\right]$ has a homogeneous regular sequence consisting of two elements of degree 2 , one of degree 3 , and one of degree 6 such that the numerator of the Hilbert series has degree at most 14. Then the Lie calculation confirms Equation (6.1).

\section{REFERENCES}

[AGJ90] Judith M. Arms, Mark J. Gotay, and George Jennings, Geometric and algebraic reduction for singular momentum maps, Adv. Math. 79 (1990), no. 1, 43-103.

[Avr81] Luchezar L. Avramov, Complete intersections and symmetric algebras, J. Algebra 73 (1981), no. 1, 248-263.

[Bea00] Arnaud Beauville, Symplectic singularities, Invent. Math. 139 (2000), no. 3, 541-549.

[Bec10] Tanja Becker, On the existence of symplectic resolutions of symplectic reductions, Math. Z. 265 (2010), no. 2, 343-363.

[Ben93] D. J. Benson, Polynomial invariants of finite groups, London Mathematical Society Lecture Note Series, vol. 190, Cambridge University Press, Cambridge, 1993.

[Ber07] Rolf Berndt, Representations of linear groups, Vieweg, Wiesbaden, 2007, An introduction based on examples from physics and number theory.

[BH93] Winfried Bruns and Jürgen Herzog, Cohen-Macaulay rings, Cambridge Studies in Advanced Mathematics, vol. 39, Cambridge University Press, Cambridge, 1993.

[BK12] Gwyn Bellamy and Toshiro Kuwabara, On deformation quantizations of hypertoric varieties, Pacific J. Math. 260 (2012), no. 1, 89-127.

[BLLT17] Michael Bulois, Christian Lehn, Manfred Lehn, and Ronan Terpereau, Towards a symplectic version of the Chevalley restriction theorem, Compos. Math. 153 (2017), no. 3, 647-666.

[Bou87] Jean-François Boutot, Singularités rationnelles et quotients par les groupes réductifs, Invent. Math. 88 (1987), no. 1, 65-68.

[Bry03] Ranee Brylinski, Dixmier algebras for classical complex nilpotent orbits via KraftProcesi models. I, The orbit method in geometry and physics (Marseille, 2000), Progr. Math., vol. 213, Birkhäuser Boston, Boston, MA, 2003, pp. 49-67.

[BS16] Gwyn Bellamy and Travis Schedler, Symplectic resolutions of quiver varieties and character varieties, arXiv:1602.00164 [math.AG].

[BS18] Gwyn Bellamy and Travis Schedler, On symplectic resolutions and factoriality of hamiltonian reductions, arXiv:1809.04301 [math.AG].

[Bul18] Michaël Bulois, On the normality of the null-fiber of the moment map for $\theta$ - and tori representations, J. Algebra 507 (2018), 502-524.

[Che55] Claude Chevalley, Invariants of finite groups generated by reflections, Amer. J. Math. 77 (1955), 778-782.

[CHS16] Joshua Cape, Hans-Christian Herbig, and Christopher Seaton, Symplectic reduction at zero angular momentum, J. Geom. Mech. 8 (2016), no. 1, 13-34.

[CLS11] David A. Cox, John B. Little, and Henry K. Schenck, Toric varieties, Graduate Studies in Mathematics, vol. 124, American Mathematical Society, Providence, RI, 2011.

[DH15] Brent Doran and Victoria Hoskins, Algebraic symplectic analogues of additive quotients, arXiv:1512.07513 [math.AG]. 
[DK02] Harm Derksen and Gregor Kemper, Computational invariant theory, Invariant Theory and Algebraic Transformation Groups, I, Springer-Verlag, Berlin, 2002, Encyclopaedia of Mathematical Sciences, 130.

[Dré04] Jean-Marc Drézet, Luna's slice theorem and applications, Algebraic group actions and quotients, Hindawi Publ. Corp., Cairo, 2004, hal-00742479, pp. 39-89.

[Eis95] David Eisenbud, Commutative algebra, Graduate Texts in Mathematics, vol. 150, Springer-Verlag, New York, 1995, With a view toward algebraic geometry.

[FHS13] Carla Farsi, Hans-Christian Herbig, and Christopher Seaton, On orbifold criteria for symplectic toric quotients, SIGMA Symmetry Integrability Geom. Methods Appl. 9 (2013), Paper 032, 18.

[FL81] William Fulton and Robert Lazarsfeld, Connectivity and its applications in algebraic geometry, Algebraic geometry (Chicago, Ill., 1980), Lecture Notes in Math., vol. 862, Springer, Berlin-New York, 1981, pp. 26-92.

[Fle81] Hubert Flenner, Rationale quasihomogene Singularitäten, Arch. Math. (Basel) 36 (1981), no. 1, 35-44.

[Fle88] Extendability of differential forms on nonisolated singularities, Invent. Math. 94 (1988), no. 2, 317-326.

[Fu03] Baohua Fu, Symplectic resolutions for nilpotent orbits, Invent. Math. 151 (2003), no. 1, $167-186$.

[Fu06] - A survey on symplectic singularities and symplectic resolutions, Ann. Math. Blaise Pascal 13 (2006), no. 2, 209-236.

[GM88] Mark Goresky and Robert MacPherson, Stratified Morse theory, Ergebnisse der Mathematik und ihrer Grenzgebiete (3) [Results in Mathematics and Related Areas (3)], vol. 14, Springer-Verlag, Berlin, 1988.

[Gor90] N. L. Gordeev, Coranks of elements of linear groups and the complexity of algebras of invariants, Algebra i Analiz 2 (1990), no. 2, 39-64; translation in Leningrad Math. J. 2 (1991), no. 2, 245-267.

[GW78] Shiro Goto and Keiichi Watanabe, On graded rings. I, J. Math. Soc. Japan 30 (1978), no. 2, 179-213.

[Har77] Robin Hartshorne, Algebraic geometry, Springer-Verlag, New York-Heidelberg, 1977, Graduate Texts in Mathematics, No. 52.

[HHS15] Hans-Christian Herbig, Daniel Herden, and Christopher Seaton, On compositions with $x^{2} /(1-x)$, Proc. Amer. Math. Soc. 143 (2015), no. 11, 4583-4596.

[HIP09] Hans-Christian Herbig, Srikanth B. Iyengar, and Markus J. Pflaum, On the existence of star products on quotient spaces of linear Hamiltonian torus actions, Lett. Math. Phys. 89 (2009), no. 2, 101-113.

[HS13] Hans-Christian Herbig and Gerald W. Schwarz, The Koszul complex of a moment map, J. Symplectic Geom. 11 (2013), no. 3, 497-508.

[HS14] Hans-Christian Herbig and Christopher Seaton, The Hilbert series of a linear symplectic circle quotient, Exp. Math. 23 (2014), no. 1, 46-65.

[HS15] - An impossibility theorem for linear symplectic circle quotients, Rep. Math. Phys. 75 (2015), no. 3, 303-331.

[HSS15] Hans-Christian Herbig, Gerald W. Schwarz, and Christopher Seaton, When is a symplectic quotient an orbifold?, Adv. Math. 280 (2015), 208-224.

[Hun98] Craig Huneke, Tight closure, parameter ideals, and geometry, Six lectures on commutative algebra (Bellaterra, 1996), Progr. Math., vol. 166, Birkhäuser, Basel, 1998, pp. $187-239$.

[Hun07] Lectures on local cohomology, Interactions between homotopy theory and algebra, Contemp. Math., vol. 436, Amer. Math. Soc., Providence, RI, 2007, Appendix 1 by Amelia Taylor, pp. 51-99.

[Ish14] Shihoko Ishii, Introduction to singularities, Springer, Tokyo, 2014.

[KKV89] Friedrich Knop, Hanspeter Kraft, and Thierry Vust, The Picard group of a G-variety, Algebraische Transformationsgruppen und Invariantentheorie, DMV Sem., vol. 13, Birkhäuser, Basel, 1989, pp. 77-87.

[KLS06] D. Kaledin, M. Lehn, and Ch. Sorger, Singular symplectic moduli spaces, Invent. Math. 164 (2006), no. 3, 591-614.

[KM98] János Kollár and Shigefumi Mori, Birational geometry of algebraic varieties, Cambridge Tracts in Mathematics, vol. 134, Cambridge University Press, Cambridge, 1998, With 
the collaboration of C. H. Clemens and A. Corti, Translated from the 1998 Japanese original.

[KP79] Hanspeter Kraft and Claudio Procesi, Closures of conjugacy classes of matrices are normal, Invent. Math. 53 (1979), no. 3, 227-247.

[KP82] On the geometry of conjugacy classes in classical groups, Comment. Math. Helv. 57 (1982), no. 4, 539-602.

[KS01] Hanspeter Kraft and Gerald W. Schwarz, Rational covariants or reductive groups and homaloidal polynomials, Math. Res. Lett. 8 (2001), no. 5-6, 641-649.

[LR79] D. Luna and R. W. Richardson, A generalization of the Chevalley restriction theorem, Duke Math. J. 46 (1979), no. 3, 487-496.

[Lun72] Domingo Luna, Sur les orbites fermées des groupes algébriques réductifs, Invent. Math. 16 (1972), 1-5.

[Lun73] Slices étales, Sur les groupes algébriques, Soc. Math. France, Paris, 1973, pp. 81-105. Bull. Soc. Math. France, Paris, Mémoire 33.

[Lun75] D. Luna, Adhérences d'orbite et invariants, Invent. Math. 29 (1975), no. 3, 231-238.

[LV73] D. Luna and Th. Vust, Un théorème sur les orbites affines des groupes algébriques semi-simples, Ann. Scuola Norm. Sup. Pisa (3) 27 (1973), 527-535 (1974).

[Mat60] Yozô Matsushima, Espaces homogènes de Stein des groupes de Lie complexes, Nagoya Math. J 16 (1960), 205-218.

[Mat89] Hideyuki Matsumura, Commutative ring theory, second ed., Cambridge Studies in Advanced Mathematics, vol. 8, Cambridge University Press, Cambridge, 1989, Translated from the Japanese by M. Reid.

[MN14] Kevin McGerty and Thomas Nevins, Derived equivalence for quantum symplectic resolutions, Selecta Math. (N.S.) 20 (2014), no. 2, 675-717.

[Nam01] Yoshinori Namikawa, Extension of 2-forms and symplectic varieties, J. Reine Angew. Math. 539 (2001), 123-147.

[Pan91] D. I. Panyushev, Rationality of singularities and the Gorenstein property of nilpotent orbits, Funktsional. Anal. i Prilozhen. 25 (1991), no. 3, 76-78.

[PS85] Claudio Procesi and Gerald Schwarz, Inequalities defining orbit spaces, Invent. Math. 81 (1985), no. 3, 539-554.

[PV94] V. L. Popov and È. B. Vinberg, Invariant theory, Algebraic geometry. IV, Encyclopaedia of Mathematical Sciences, vol. 55, Springer-Verlag, Berlin, 1994, Linear algebraic groups. Invariant theory, A translation of it Algebraic geometry. 4 (Russian), Akad. Nauk SSSR Vsesoyuz. Inst. Nauchn. i Tekhn. Inform., Moscow, 1989, Translation edited by A. N. Parshin and I. R. Shafarevich, pp. vi+284.

[Rei80] Miles Reid, Canonical 3-folds, Journées de Géometrie Algébrique d'Angers, Juillet 1979/Algebraic Geometry, Angers, 1979, Sijthoff \& Noordhoff, Alphen aan den RijnGermantown, Md., 1980, pp. 273-310.

[Sch80] Gerald W. Schwarz, Lifting smooth homotopies of orbit spaces, Inst. Hautes Études Sci. Publ. Math. (1980), no. 51, 37-135.

[Sch89] - The topology of algebraic quotients, Topological methods in algebraic transformation groups (New Brunswick, NJ, 1988), Progr. Math., vol. 80, Birkhäuser Boston, Boston, MA, 1989, pp. 135-151.

[Sch95] _ Lifting differential operators from orbit spaces, Ann. Sci. École Norm. Sup. (4) 28 (1995), no. 3, 253-305.

[SGA1] Revêtements étales et groupe fondamental (SGA 1), Documents Mathématiques (Paris) [Mathematical Documents (Paris)], vol. 3, Société Mathématique de France, Paris, 2003, Séminaire de géométrie algébrique du Bois Marie 1960-61. [Algebraic Geometry Seminar of Bois Marie 1960-61], Directed by A. Grothendieck, With two papers by M. Raynaud, Updated and annotated reprint of the 1971 original [Lecture Notes in Math., 224, Springer, Berlin]

[SL91] Reyer Sjamaar and Eugene Lerman, Stratified symplectic spaces and reduction, Ann. of Math. (2) 134 (1991), no. 2, 375-422.

[ST54] G. C. Shephard and J. A. Todd, Finite unitary reflection groups, Canadian J. Math. 6 (1954), 274-304.

[Sta] Jason Starr, The homology groups of the smooth locus of a singular variety, https://mathoverflow.net/questions/309931/. 
[Sta78] Richard P. Stanley, Hilbert functions of graded algebras, Advances in Math. 28 (1978), no. $1,57-83$.

[Sta79] Invariants of finite groups and their applications to combinatorics, Bull. Amer. Math. Soc. (N.S.) 1 (1979), no. 3, 475-511.

[Sta18] The Stacks Project Authors, Stacks Project, https://stacks.math.columbia.edu, 2018.

[Ter12] Ronan Terpereau, Schémas de hilbert invariants et théorie classique des invariants, 2012, Thesis (Ph.D.)-Université de Grenoble.

[Ter14] - Invariant Hilbert schemes and desingularizations of symplectic reductions for classical groups, Math. Z. 277 (2014), no. 1-2, 339-359.

[Ver00] Misha Verbitsky, Holomorphic symplectic geometry and orbifold singularities, Asian J. Math. 4 (2000), no. 3, 553-563.

[Vin86] È. B. Vinberg, Complexity of actions of reductive groups, Funktsional. Anal. i Prilozhen. 20 (1986), no. 1, 1-13, 96.

[vLCL92] M. A. A. van Leeuwen, A. M. Cohen, and B. Lisser, Lie, a package for lie group computations, Computer Algebra Nederland, Amsterdam. Available at http://wwwmathlabo.univ-poitiers.fr/ maavl/LiE/, 1992.

[Wat74] Keiichi Watanabe, Certain invariant subrings are Gorenstein. I, II, Osaka J. Math. 11 (1974), 1-8; ibid. 11 (1974), 379-388.

[Wat83] , Rational singularities with $k^{*}$-action, Commutative algebra (Trento, 1981), Lecture Notes in Pure and Appl. Math., vol. 84, Dekker, New York, 1983, pp. 339-351.

[Weh92] David L. Wehlau, A proof of the Popov conjecture for tori, Proc. Amer. Math. Soc. 114 (1992), no. 3, 839-845.

[Wey39] Hermann Weyl, The Classical Groups. Their Invariants and Representations, Princeton University Press, Princeton, N.J., 1939

[Wig59] Eugene P. Wigner, Group theory: And its application to the quantum mechanics of atomic spectra, Expanded and improved ed. Translated from the German by J. J. Griffin. Pure and Applied Physics. Vol. 5, Academic Press, New York, 1959.

E-mail address: herbighc@gmail.com

Departamento de Matemática Aplicada, Universidade Federal do Rio de Janeiro, Av. Athos da Silveira Ramos 149, Centro de Tecnologia - Bloco C, CEP: 21941-909 - Rio de JANEIro, Brazil

E-mail address: schwarz@brandeis.edu

Department of Mathematics, Brandeis University, Waltham, MA 02454-9110, USA

E-mail address: seatonc@rhodes.edu

Department of Mathematics and Computer Science, Rhodes College, 2000 N. ParkWAY, Memphis, TN 38112, USA 